\title{
Structure and growth of tetracene on $\operatorname{Ag(111)}$
}

\author{
S. Soubatch, ${ }^{*}$ I. Kröger, C. Kumpf, and F. S. Tautz \\ Peter Grünberg Institut (PGI-3), Forschungszentrum Jülich, 52425 Jülich, Germany and \\ Jülich Aachen Research Alliance (JARA)—Fundamentals of Future Information Technology, 52425 Jülich, Germany
}

(Received 4 July 2011; published 9 November 2011)

\begin{abstract}
The structure of the tetracene/ $\mathrm{Ag}(111)$ interface in the coverage range $\theta=0$ to $2.4 \mathrm{ML}$ is studied with scanning tunneling microscopy (STM) at $8 \mathrm{~K}$ and with low energy electron diffraction (LEED) at $\mathrm{T}=300 \ldots$ $100 \mathrm{~K}$. For $\theta \lesssim 0.01 \mathrm{ML}$, one-dimensional (1D) diffusion of single molecules along $\langle 01 \overline{1}\rangle$-directions is observed even at $8 \mathrm{~K}$. For $0.1 \mathrm{ML}<\theta<0.5 \mathrm{ML}$ molecules are homogeneously distributed over the surface forming a disordered phase (static at $\mathrm{T}=8 \mathrm{~K}$, dynamic at $\mathrm{T}=25 \mathrm{~K}$ ), indicating a repulsive intermolecular interaction ( $\delta$-phase). For $\theta \gtrsim 0.5 \mathrm{ML}$, local ordering in the commensurate $\gamma$-phase is observed. Further increase of the coverage yields a compressed monolayer (ML) phase $(\theta \equiv 1 \mathrm{ML})$ with point-on-line registry ( $\alpha$-phase). The interaction between molecules has been calculated with the force-field approach to rationalize the molecular packing motifs in the various phases. Under most circumstances molecule-molecule interactions are repulsive, in agreement with experimental findings. A simulation of the adsorption up to $\theta=1 \mathrm{ML}$ according to the random sequential adsorption (RSA) algorithm shows that the disorder-to-order transition from the $\delta$ - to $\gamma$-phase occurs close to random close packing (RCP), $\theta=0.5-0.6 \mathrm{ML}$. Since tetracene molecules are a two-dimensional (2D) representation of Onsager's hard rod model, this suggests that this phase transition is driven both energetically and entropically. For $\theta \approx 2.23 \mathrm{ML}$ a metastable bilayer phase with point-on-line coincidence is observed ( $\beta$-phase). The basic structural unit of this phase is a triplet of molecules that are tilted along the long molecular axis against each other; at least one of these molecules is tilted out of the surface plane. Within the $\beta$-phase a superstructure of alternating rotation domains is observed. This superstructure has a period of $7.4 \mathrm{~nm}$. The molecular packing in the $\beta$-phase resembles the packing in the bulk crystal structure of tetracene, its formation can therefore be interpreted as incipient pseudomorphic growth of tetracene on $\mathrm{Ag}(111)$. However, pseudomorphic growth cannot be continued beyond the $\beta$-phase.
\end{abstract}

DOI: 10.1103/PhysRevB.84.195440

PACS number(s): 68.65.-k, 68.37.Ef, 68.43.Fg

\section{INTRODUCTION}

Tetracene $\left(\mathrm{C}_{18} \mathrm{H}_{12}\right)$, abbreviated as Tc from now on, consisting of four fused benzene rings [cf. inset in Fig. 1(b)], is an organic semiconductor. With applications of organic semiconductors in organic (opto-)electronics in mind, the growth of organic semiconductors on various inorganic substrates has been studied in recent years. ${ }^{1-8}$ In these studies it has turned out that often the growth of organic semiconductor films exhibits certain features that are not found in inorganic epitaxy (e.g., Refs. 1,2,8, and 9). This has triggered a general interest in organic film growth and in the formation of order at the interfaces between organic semiconductors and, e.g., metals. Here we present a comprehensive investigation of a model system that highlights a range of special features of ordering and growth of molecules on metals. We believe that many of the results presented here for the case of tetracene on $\mathrm{Ag}(111)$ are representative and therefore mutatis mutandis transferable to other molecule/metal systems.

The peculiarities of ordering and growth of organics at metal surfaces stem from the special properties of the molecular building blocks. For example, organic molecules are extended objects with linear dimensions that are typically larger than the in-plane lattice constants of the substrate. On adsorption at an inorganic surface, a typical organic semiconductor molecule occupies (and thus blocks) not only one but several adsorption sites. The shape anisotropy of organic semiconductor molecules implies that the molecule can adsorb in various orientations, both in-plane and out-of-plane. Typical semiconductor molecules bond weakly to metal surfaces with a dominant contribution to the binding energy from the unspecific dispersion interaction. Common adsorption energies are therefore below $1 \mathrm{eV}$ per molecule., ${ }^{1,10,11}$ The weak and unspecific molecule-substrate interaction implies low diffusion and incorporation barriers and thus will affect the growth kinetics of organic semiconductor films on metals. The interaction between the molecules is also nonchemical, giving rise to considerable structural flexibility of the growing film. In some cases one even observes repulsive interaction between adsorbed molecules, ${ }^{2,9,12-14}$ making classical nucleation theory incapable of describing the behavior of the adsorption layer at coverages below one monolayer (ML).

The consequences of these special properties are manifold. For instance, the large size of organic semiconductor molecules and generally weak interactions give rise to new forms of registry (point-on-line, line-on-line) at the interface, besides the more common ones (commensurate, incommensurate)..$^{7,15-19}$ The anisotropic shape of molecules should favor the role of entropic and topological effects in the emergence of order. ${ }^{8,20}$ Also, as the film grows in thickness, one may expect the molecular orientation to evolve from the flat adsorption enforced by the substrate to tilted arrangements caused by intermolecular interactions. Indeed, a special form of pseudomorphic growth, in which not only the lattice structure but also the molecular orientation changes as compared to the native bulk structure of the organic crystal, appears possible as a result of competing molecule-molecule and molecule-substrate interactions. In this context the unusual 

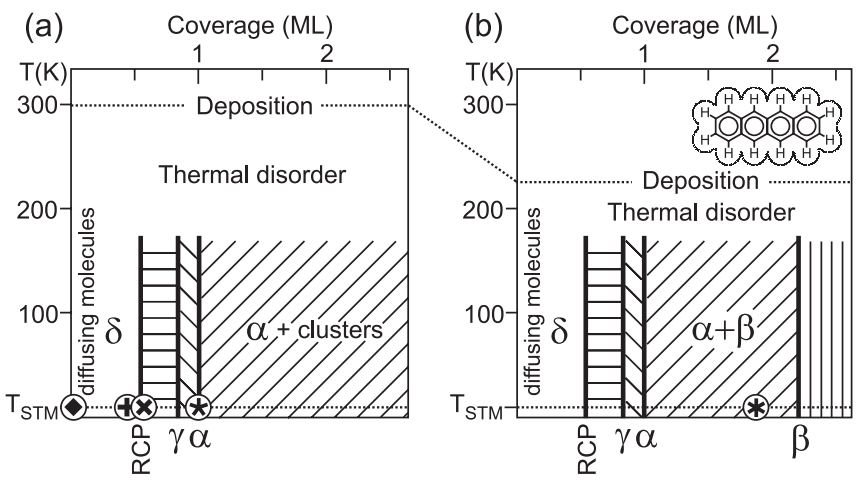

FIG. 1. Phase diagrams of $\mathrm{Tc} / \operatorname{Ag}(111)$ after deposition at (a) room temperature and (b) low temperature $(\mathrm{T} \leqslant 230 \mathrm{~K})$. Vertical bars denote experimentally observed $\alpha, \beta$, and $\gamma$ phases and the estimated coverage of the RCP layer. Hatched areas indicate phase coexistence. High temperature boarders for all phases are approximated. Points for which STM data are shown in this article are marked with $\bullet, \mathbf{+}$, $\boldsymbol{*}, \star$, and $\boldsymbol{*}$. Inset in (b): chemical structure of Tc.

desorption behavior of benzene on $\mathrm{Ru}(001)^{21}$ and bithiophene on $\mathrm{Cu}(110)^{22}$ should be noted: The third layer desorbs at lower temperatures than the multilayer, indicating that due to structural modification by the substrate the third layer becomes less stable than the bulk. Similar processes were also observed for bigger molecules. ${ }^{3}$ The complexity of organic film growth is further enhanced by the well-known polymorphism of organic materials, which can be reflected by the (co-)existence of multiple thin film phases at surfaces. ${ }^{23-28}$

In spite of the previously mentioned complexities, the general theory of crystal growth can still be applied to organic film growth after appropriate revision. ${ }^{3-6,29-32}$ Its adaption to organic systems is an ongoing project, requiring input from carefully chosen experiments that help to single out the special features of ordering and growth at the organic/metal interface. We have chosen $\mathrm{Tc} / \mathrm{Ag}(111)$ as a suitable model system because tetracene is a representative of the class of polycyclic $\pi$-conjugated platelet molecules. The Tc molecule is structurally rigid, has a simple 2:1 shape anisotropy ${ }^{1}$ [cf. inset in Fig. 1(b)], exhibits a bulk crystal structure in which molecules are not co-planar, lacks functional groups, and interacts weakly with $\mathrm{Ag}(111)$. All of this makes $\mathrm{Tc} / \mathrm{Ag}(111)$ an ideal model system for the purpose of this paper. Indeed, the $\mathrm{Tc} / \mathrm{Ag}(111)$ interface reveals an interesting structural phase diagram. ${ }^{1}$ Yet the available experimental data on this interface is limited. ${ }^{1,10,33-35}$ The emphasis in the present study is placed on structural data collected with scanning tunneling microscopy (STM) and low energy electron diffraction (LEED); in particular we present a refined phase diagram of the $\mathrm{Tc} / \mathrm{Ag}(111)$ interface in the coverage range 0 to $2.4 \mathrm{MLs}$. Based on the structural data presented here, electronic structure studies, ${ }^{35}$ kinetic growth studies, etc., may be performed in the future.

The paper is organized as follows. In Sec. II a short summary of experimental methods is given. In Sec. III we discuss phases and phase transformations at coverages less than or equal to a full ML: The study of adsorption at very low coverages (Sec. III A) allows conclusions regarding Tc diffusion on $\mathrm{Ag}(111)$, while for coverages from 0.5 to $1 \mathrm{ML}$ the hierarchy of ordered phases ( $\delta$-phase in Sec. III B, $\gamma$-phase in Sec. III C, and $\alpha$-phase in Sec. III E) and the driving forces behind the structural phase transformations can be analyzed (Secs. III D and IIIF). In Sec. IV the complex structure of the $\beta$-phase (Sec. IV A) is studied with high resolution STM (Sec. IV B), and a structural model is developed (Sec. IV C). This analysis allows conclusions regarding pseudomorphic growth at metal-organic interfaces (Sec. IV D and IV E). The paper closes with a short conclusion (Sec. V).

\section{EXPERIMENTAL METHODS}

All experiments were carried out in an ultra-high vacuum chamber with a base pressure below $10^{-10}$ mbar. To deposit Tc, we evaporated molecules (Sigma-Aldridge) from a homemade effusion cell onto the $\operatorname{Ag}(111)$ single crystal substrate. The substrate was cleaned by a series of sputtering $\left[\mathrm{p}\left(\mathrm{Ar}^{+}\right)=\right.$ $4 \times 10^{-5}$ mbar, $\left.\mathrm{U}=800 \mathrm{~V}\right]$ and annealing $(800 \mathrm{~K})$ cycles. The crystal temperature during molecule deposition $T_{\text {dep }}$ was varied in different experiments from $210 \mathrm{~K}$ to $300 \mathrm{~K}$ in order to obtain different phases of $\mathrm{Tc}$ on $\operatorname{Ag}(111)^{1}$ (for an overview of the phase diagram, see Fig. 1). The deposition rate was calibrated by means of x-ray photoelectron spectroscopy (XPS). ${ }^{36}$ A conventional LEED optics was used for identifying Tc phases after deposition at $T_{\text {dep }}$ and subsequent cooling to below the disorder-to-order transition temperature $T_{\mathrm{tr}}$, which is approximately $180 \mathrm{~K}$. The molecular arrangement of $\mathrm{Tc}$ in the various phases and the local electronic properties of individual molecules in these phases were studied with a low temperature scanning tunneling microscope at $T_{\mathrm{STM}}=8 \mathrm{~K}$. In the phase diagram of Fig. 1 and throughout the paper we quote Tc surface coverages in ML. 1 ML refers to the $\alpha$-phase with a Tc surface density of $1.0 \times 10^{14}$ molecules $/ \mathrm{cm}^{2}$ (cf. Table I).

\section{PHASES WITH $\theta \leqslant 1$ ML}

All phases discussed in this section have been prepared by depositing Tc molecules at $300 \mathrm{~K}$, followed by slow postdeposition cooling to $150 \mathrm{~K}$ where LEED has been done to confirm the presence or absence of order. Then the sample was transferred into the STM and images were recorded at $T_{\mathrm{STM}}=8 \mathrm{~K}$. This procedure guarantees that we are looking at thermodynamically stable phases.

\section{A. Diffusing molecules $(\theta \ll 1 \mathrm{ML}, \mathrm{T}=8 \mathrm{~K})$}

At extremely low coverage $\theta<0.01 \mathrm{ML}$, we observe in the STM a number of bright stripes oriented along $\langle 01 \overline{1}\rangle$-directions of the substrate and crossing each other (Fig. 2). We never observed single immobilized molecules or (immobile) islands of molecules, except for molecules decorating step edges. The stripes on terraces can be interpreted as traces along which Tc molecules diffuse back and forth with speeds that are much faster than the scanning speed of the microscope. The molecular motion along $\langle 01 \overline{1}\rangle$-directions can be observed directly in tunneling current vs time spectra recorded above a diffusion trace. This reveals a typical "telegraph noise" pattern of the tunneling current [Fig. 2(b)], with high (low) current values corresponding to the presence (absence) of the diffusing molecule beneath the tip. The fact that even at temperatures below $10 \mathrm{~K}$ the barrier for surface diffusion along 
TABLE I. Ordered and disordered phases of Tc/Ag(111). Unit cell vectors and angles for $\alpha$ - and $\gamma$-phases are derived from STM.

\begin{tabular}{|c|c|c|c|c|c|c|c|}
\hline Phase & Unit Cell & Type & $\begin{array}{l}\text { Number of Molecules } \\
\text { in Unit Cell }\end{array}$ & $\begin{array}{l}\text { Unit Cell } \\
\text { Area }\left(\AA^{2}\right)\end{array}$ & $\begin{array}{c}\text { Area per } \\
\text { Molecule }\left(\AA^{2}\right)\end{array}$ & $\begin{array}{c}\text { Surface } \\
\text { Density }\left(\mathrm{cm}^{-2}\right)\end{array}$ & $\begin{array}{l}\text { Coverage } \\
(\mathrm{ML} \text { of } \alpha)\end{array}$ \\
\hline mobile molecules & & 1D surface diffusion & & & & $\leqslant 10^{12}$ & $\leqslant 0.01$ \\
\hline$\delta$ & & disordered layer & & & & $<5 \times 10^{13}$ & $<0.5$ \\
\hline $\mathrm{RCP}$ & & disordered layer & & & & $5-6 \times 10^{13}$ & $0.5-0.6$ \\
\hline & $\begin{array}{c}\left|\mathbf{g}_{1}\right|=13 \AA \\
\left|\mathbf{g}_{2}\right|=13 \AA \\
\gamma=135^{\circ}\end{array}$ & & & & & & \\
\hline$\gamma$ & $\begin{array}{c}\text { (ideal commensurate: } \\
\left|\mathbf{g}_{1}\right|=12.6 \AA \\
\left|\mathbf{g}_{2}\right|=12.6 \AA \\
\left.\gamma=133.14^{\circ}\right) \\
\left|\mathbf{a}_{1}\right|=13.1 \AA \\
\left|\mathbf{a}_{2}\right|=8.2 \AA \\
\alpha=87^{\circ}\end{array}$ & commensurate & 1 & 121 & 121 & $8.3 \times 10^{13}$ & 0.83 \\
\hline$\alpha$ & $\begin{array}{l}\text { (derived from SPA-LEED }{ }^{1} \text { : } \\
\qquad \begin{array}{c}\left|\mathbf{a}_{1}\right|=12.9 \AA \\
\left|\mathbf{a}_{2}\right|=7.8 \AA \\
\left.\alpha=83.2^{\circ}\right) \\
\left|\mathbf{b}_{1}\right|=14 \AA\end{array}\end{array}$ & point-on-line & 1 & 100 & 100 & $10 \times 10^{13}$ & 1 \\
\hline$\beta$ & $\begin{aligned}\left|\mathbf{b}_{2}\right| & =74 \AA \\
\beta & =72^{\circ}\end{aligned}$ & point-on-line & 22 & 985 & 44.8 & $22.3 \times 10^{13}$ & 2.23 \\
\hline
\end{tabular}

(a)
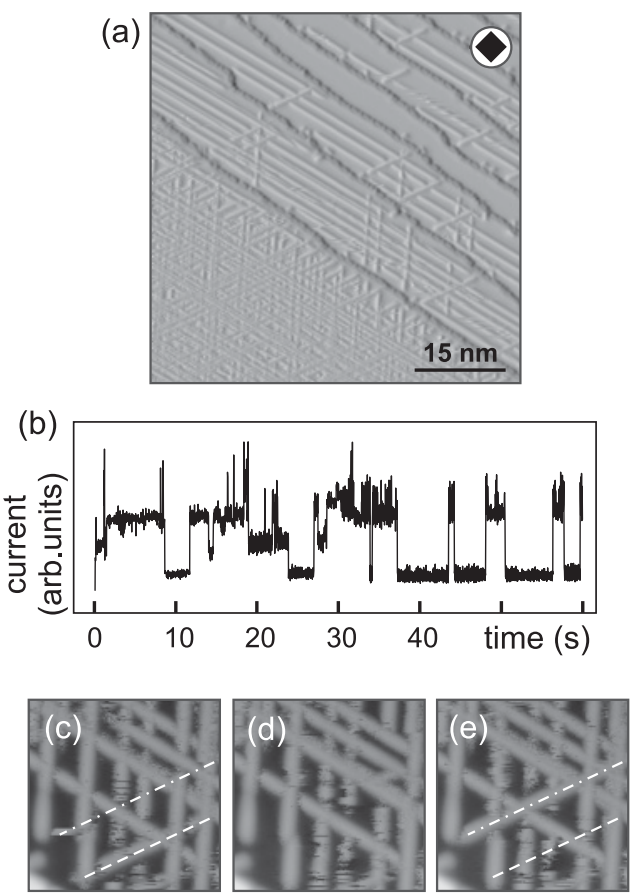

FIG. 2. (a) Surface mobility of $\mathrm{Tc}$ molecules on $\operatorname{Ag}(111)$ at $8 \mathrm{~K}$. The STM image was recorded with $0.4 \mathrm{~V}$ bias voltage and $95 \mathrm{pA}$ tunneling current (A shadow filter was applied for improved contrast $\left.{ }^{68}\right)$. The stripes represent the traces of moving Tc molecules. Single immobilized Tc molecules can be recognized at the step edges of the substrate. The symbol indicates the point in the phase diagram of Fig. 1 that corresponds to the STM image. (b) Time spectrum of the tunneling current recorded above a diffusion trace. (c)-(e) Subsequent STM images of the same sample area, recorded with $0.08 \mathrm{~V}$ bias voltage and $95 \mathrm{pA}$ tunneling current. Dashed lines mark diffusion traces of molecules which leave the image frame (dash line) or appear (dash-dot line). $\langle 01 \overline{1}\rangle$-directions can be overcome reveals a rather weak interaction at the interface. A similar behavior and corresponding STM images have been observed for pentacene on the $\operatorname{Ag}(111)$ surface. ${ }^{37}$ In contrast, diffusion of pentacene on more reactive surfaces, e.g., $\mathrm{Cu}(111)$, at low temperature $(<10 \mathrm{~K})$ is known to demand additional activation by the STM tip. ${ }^{38}$

In an image series recorded on wide $\operatorname{Ag}(111)$ terraces, some Tc traces disappear and in a later image appear again, demonstrating the ability of molecules to cover long distances and escape from the scanning window [Figs. 2(c)-2(e)]. Since each observed trace is assigned to a molecule moving over a distance longer than the scanning range, we cannot use the number of traces directly for estimating the average surface density of Tc. However, one clearly sees that step-edges of silver terraces are not permeable for Tc diffusion [diffusion traces terminate at step edges, cf. Fig. 2(a)]. Correspondingly, narrow terraces exhibit a much smaller density of transverse traces, i.e., traces that are oriented nearly perpendicular to the step edges, although the flux of molecules arriving at the surface is homogeneous over entire substrate irrespective of the local terrace size. Assuming that the adsorption rate does not depend on the terrace width and that the probability for a molecule to adopt either of the three possible $\langle 01 \overline{1}\rangle$-directions upon adsorption is the same $(1 / 3)$, we can estimate the mean coverage by counting the number of transverse traces on a narrow terrace. This yields a density of approximately $16 \times$ $10^{11}$ molecules $/ \mathrm{cm}^{2}$.

\section{B. Disordered $\delta$-phase $(\theta<1$ ML, T $<180 \mathrm{~K})$}

At larger coverages $(0.1 \mathrm{ML}<\theta<0.5 \mathrm{ML})$, molecules are homogeneously distributed over the surface [e.g., Fig. 3(a), Tc surface density $4.4 \times 10^{13}$ molecules $/ \mathrm{cm}^{2}$ ], forming a static (at $T_{\mathrm{STM}}$ ) disordered phase. Correspondingly, no diffraction spots are observed in LEED, in agreement with previously 
(a)

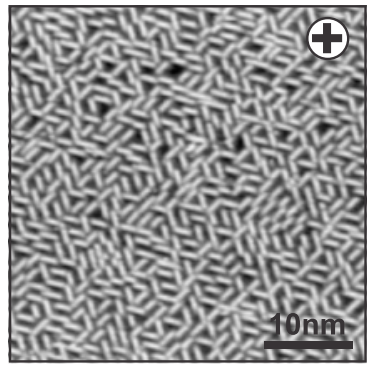

(c)

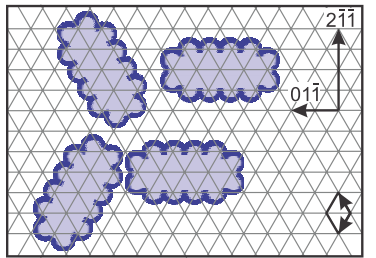

(b)

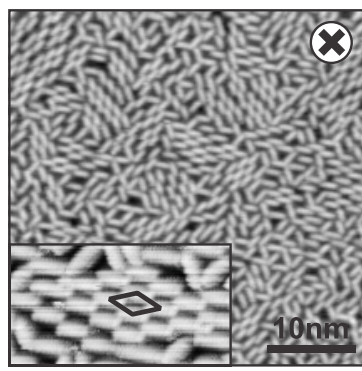

(d)

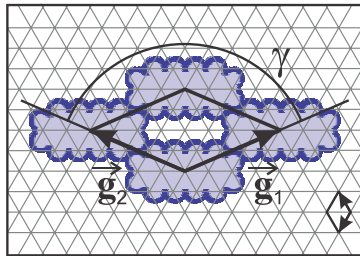

FIG. 3. (Color) (a), (b) STM images of Tc on $\mathrm{Ag}(111)$ at different conditions corresponding to points $\boldsymbol{+}$ and $\boldsymbol{x}$ in the phase diagram of Fig. 1. Tc surface densities (a) $4.4 \AA \times 10^{13}$ and (b) $5.6 \AA \times 10^{13} \mathrm{~cm}^{-2}$. Inset in (b): Small domain of $\gamma$-phase. (c) Typical arrangement of Tc molecules at coverages less than RCP. (d) Unit cell of the $\gamma$-phase.

reported results. ${ }^{1}$ In some experiments we kept the sample surface at $230 \mathrm{~K}$ for 15 hours (after deposition at $T_{\text {dep }}=$ $300 \mathrm{~K}$ ) before slowly cooling to $8 \mathrm{~K}$; still, disordered layers without any evidence for local ordering were observed. We can thus conclude that in the given parameter range the disorder at the $\mathrm{Tc} / \mathrm{Ag}(111)$ interface is not merely caused by kinetic barriers but indeed thermodynamically favorable. We refer to the disordered phase as the $\delta$-phase.

The $\delta$-phase is characterized by a strict orientation of Tc molecules along one of the $\langle 01 \overline{1}\rangle$-directions of the substrate, a tendency to local arrangements in which two or three molecules-each aligned with one of the $\langle 01 \overline{1}\rangle$-directionsmeet head on [Fig. 3(c)] and an interlocking of the molecules which suppresses the rotational and translational mobility of individual Tc molecules. Factors that favor this kind of disorder are a lack of attraction or even repulsion between Tc molecules, a molecule-substrate interaction that is sufficiently strong to enforce planar adsorption and an orientation along $\langle 01 \overline{1}\rangle$-directions, and the strongly anisotropic cigar-like shape of the Tc molecule in conjunction with the orientational anchoring. Details will be discussed in Sec. III D.

Already a slight thermal activation converts the static disorder (described in the previous paragraph) into a dynamic one: At $25 \mathrm{~K}$, the detailed local arrangement of Tc molecules changes from scan to scan; the general appearance of the disorder pattern, however, remains unchanged. At the same time, scans at $25 \mathrm{~K}$ are more noisy and unstable, which directly indicates molecular displacements and/or reorientations within the interface layer. It is difficult to decide whether at $25 \mathrm{~K}$ the layer on its own, i.e., without the influence of the STM tip, would exhibit static or dynamic disorder. But certainly at $25 \mathrm{~K}$ the layer is more susceptible to the influence of the tip than at $8 \mathrm{~K}$, even at the very low tunneling currents used in our experiments $(<0.1 \mathrm{nA})$. Extrapolating this behavior to larger temperatures, one may expect that close to the deposition temperature $(230 \mathrm{~K})$ the layer is a $2 \mathrm{D}$ quasiliquid in which the

local structure is constantly rearranging. ${ }^{1}$ Further, one may speculate that this quasiliquid undergoes a glass transition when cooled to $T_{\mathrm{STM}}=8 \mathrm{~K}$. If this was true, the image in Fig. 3(a) would indeed be one of a $2 \mathrm{D}$ glass.

\section{C. $\gamma$-phase: Disorder-to-order transition at $\theta<1 \mathrm{ML}, \mathrm{T}<180 \mathrm{~K}$}

A small increase of the Tc surface density from $4.4 \times$ $10^{13}$ molecules $/ \mathrm{cm}^{2}$ to $5.6 \times 10^{13}$ molecules $/ \mathrm{cm}^{2}$ leads to local ordering in the film: In Fig. 3(b) we observe small ordered domains (typically consisting of 10-20 molecules) of a chess board structure [Fig. 3(b), inset] that was not reported before. In the following we will refer to the chess board structure as the $\gamma$-phase of $\operatorname{Tc}$ on $\operatorname{Ag}(111)$. The small size of the ordered domains and the incoherence of their relative positions explain why no LEED pattern is observed. Notably, the molecular orientation in all ordered domains still coincides with the main crystallographic directions of the silver substrate, confirming once more the general tendency that $\mathrm{Tc}$ molecules align with $\langle 01 \overline{1}\rangle$-directions.

The superstructure unit cell of the $\gamma$-phase is almost ideally rhombic with a lattice constant $\left|\mathbf{g}_{1}\right| \approx\left|\mathbf{g}_{2}\right|$ of approximately 13 $\AA$ and an angle of approximately $135^{\circ}$ [Fig. 3(d)]. Measuring the separation between parallel molecules along a $\langle 2 \overline{1} \overline{1}\rangle$ direction for 100 pairs, we get a broad distribution (full width at half maximum $\mathrm{FWHM}=0.6 \AA$ ) with a maximum at $9.9 \AA$. This fits well to the length of the $\langle 2 \overline{1} \overline{1}\rangle$-translation vector of the $\mathrm{Ag}$ crystal (10.01 $\AA$ ), revealing a strong effect of the substrate on the molecular arrangement: The distance between molecules is nearly four times the distance between atom rows of silver. In the perpendicular direction $(\langle 01 \overline{1}\rangle)$ the distance between molecules is about $22.4 \AA$, i.e., close to $8 \times$ $\mathrm{a}_{111}=23.12 \AA$, suggesting the $\gamma$-phase to be commensurate. Taking into account the size of the Tc molecule $(13.7 \times$ $7 \AA^{2}$, Ref. 1), the separation between two molecules along the $\langle 2 \overline{1} \overline{1}\rangle$-directions is not enough to place another molecule in between, hence the gaps between molecules that lead to the chess boardlike appearance of the $\gamma$-phase. Note, however, that the $\langle 2 \overline{1} \overline{1}\rangle$-rows of Tc interdigitate and the molecules therefore come closer to each other at their short ends. With a unit cell area of $121 \AA^{2}$, occupied by one molecule (i.e., a surface density of $8.3 \times 10^{13}$ molecules $/ \mathrm{cm}^{2}$ ), the $\gamma$-phase is denser than the disordered $\delta$-phase in Fig. 3(a). Nevertheless, it still allows a substantial separation between molecules (especially within the $\langle 2 \overline{1} \overline{1}\rangle$-rows) and thus complies with the constraints of intermolecular repulsion (see subsequent discussion).

For surface densities about $10^{14}$ molecules $/ \mathrm{cm}^{2}$, the $\alpha$ phase appears upon post-deposition cooling. The area per molecule for the $\alpha$-phase is $100 \AA^{2}$. A detailed description of the $\alpha$-phase will be given in Sec. III E.

\section{Discussion of the phase behavior for $\theta<1 \mathrm{ML}, \mathrm{T}<180 \mathrm{~K}$}

We now turn to a discussion of the driving forces behind the structural organization in the coverage range $\theta<1 \mathrm{ML}$. Three striking observations have been reported in Secs. III A to IIIC. (1) Any form of nucleation in the submonolayer coverage regime is absent, and therefore Tc molecules are homogeneously distributed on the surface for a broad range 
of surface densities. (2) Disorder persists to almost $1 \mathrm{ML}$ Tc coverage. (3) A disorder-to-order phase transition as a function of coverage occurs in the densely packed layer at $\theta$ close to 1 ML. We designate the $\delta \rightarrow \gamma$ transformation as a phase transition as a function of $\theta$ at constant $T_{\mathrm{STM}}=8 \mathrm{~K}$, although in our experiments we have never crossed the phase boundary between the two at constant $T=T_{\mathrm{STM}}$. Rather, we enter both the statically disordered $\delta$-phase at $T_{\mathrm{STM}}$ and the layer with small patches of $\gamma$-phase at $T_{\mathrm{STM}}$ coming from the deposition temperature $T_{\mathrm{dep}}$, where the Tc layers are fluid at all coverages. However, coming from high temperature, we assume that we reach equilibrium at low temperature, i.e., we suppose that both the $\delta$ - and $\gamma$-structures that we observe at $T_{\mathrm{STM}}$ are equilibrium phases. Therefore, if we could increase the coverage at $T_{\mathrm{STM}}$ infinitely slowly, we would expect to recover the same $\delta$ - and $\gamma$-structures as observed in Figs. 3(a) and 3(c).

Three contributions to the free energy, whose minimum determines the equilibrium state, must be taken into account: the sum of all intermolecular interaction energies $E_{I}$, the sum of all interaction energies between the substrate and the Tc molecules $E_{S}$, and the total entropy of the molecular layer $S$. The molecule-substrate interaction is attractive, otherwise no adsorption would occur. It further leads to the preferential orientation of Tc molecules on the substrate and their confinement to the first layer. The absence of any form of nucleation and the homogeneous distribution of molecules over the complete surface for all $\theta<1 \mathrm{ML}$ indicates that the molecules tend to maximize their mutual distances. This points to a repulsive intermolecular interaction.

The conjecture of a repulsive Tc-Tc interaction is confirmed by the measurements of the desorption energy as a function of coverage: Gonella et al. employed intermolecular repulsion to explain the observed decrease of the desorption energy per molecule for increasing coverage. ${ }^{10} \mathrm{~A}$ possible reason for the intermolecular repulsion is the repulsion between parallel adsorption dipoles of identical molecules. These adsorption dipoles may originate either in charge transfer from the adsorbed molecules to the surface or vice versa ${ }^{10}$ or in the displacement of electron density of the metal surface by an (inert) adsorbate ("push-back" effect). ${ }^{39-41}$ Intermolecular repulsion can be also caused by Coulomb interaction between partially charged parts of molecules. ${ }^{9}$ Another mechanism, by which interadsorbate repulsion can arise, is the formation of an electronic standing wave pattern between adsorbates, as has been suggested for pentacene/Cu(110). ${ }^{42}$ This suggestion is based on the measurement of the $\mathrm{Cu}$ adatom distribution at low temperatures by Repp et al. ${ }^{43}$ that was explained in terms of Hyldgard-Persson theory. ${ }^{44}$

It should also be noted in this context that the depopulation of the Shockley surface state by the adsorbate layer that is observed for the $\alpha$-phase of Tc/Ag(111) (cf. Sec. IIIE) is of relevance both for the molecule-substrate and the moleculemolecule interactions. For example, for Xe adsorption on $\operatorname{Pt}(111)$ it has been shown that an upshift of the Pt surface state by $150 \mathrm{meV}$ leads to a repulsive contribution to the molecule-substrate interaction of $50 \mathrm{meV}$ per Xe atom. ${ }^{45} \mathrm{At}$ the same time, the local depletion of the surface-state wave function around the adsorbate lowers the Pauli repulsion for neighboring adsorbates, thus leading to an effective surfacestate mediated adsorbate-adsorbate attraction. In the case of
$\mathrm{Xe}$ on $\mathrm{Pt}$ this attraction extends over a radius of approximately $9 \AA$ and leads to island formation of Xe. In the present case of $\mathrm{Tc} / \mathrm{Ag}(111)$ we know from the absence of nucleation that the overall molecule-molecule interaction is repulsive. The surface state of $\operatorname{Ag}(111)$ contains $8.7 \times 10^{-3}$ electrons per surface atom. With its effective mass of $0.42 \mathrm{~m}_{\mathrm{e}}$ and its binding energy at the $\Gamma$-point of $-67 \mathrm{meV}$, the complete depopulation of the surface state upon Tc adsorption contributes a repulsive contribution of approximately $8 \mathrm{meV}$ per molecule to the total adsorption energy. Hence, a possible surface-state mediated attraction between Tc molecules on $\operatorname{Ag}(111)$ will be very small. However, the range of this attraction, as given by the first maximum of Friedel oscillations for $\mathrm{Ag}(111)$, is significant. It amounts to approximately $37 \AA$.

By inspection of the STM image in Figs. 3(a) and 3(c), the surface density of the $\delta$-phase appears to be close to random close packing (RCP). (For a more detailed analysis of RCP densities in Tc layers, see subsequent discussion). On the other hand the surface density of the $\gamma$-phase $(8.3 \times$ $10^{13}$ molecules $/ \mathrm{cm}^{2}$ ) is larger than that of the $\delta$-phase [in Fig. 3(a), $4.4 \times 10^{13}$ molecules $/ \mathrm{cm}^{2}$ ]. Because of the attractive interaction between the substrate and Tc molecules, which is larger than intermolecular attraction between first- and second-layer molecules, there is an energy gain in moving a molecule from the second into the first layer. Therefore, an energy gain is associated with the $\delta \rightarrow \gamma$ transition because additional molecules, instead of being loosely bound in the second layer on top of the $\delta$-phase, can move to the interface and minimize their energy by attraction with the metal substrate.

Apart from this energetic contribution, the appearance of the $\gamma$-phase may also be assisted by an increase of the total entropy at constant coverage, despite the fact that the $\gamma$-phase is ordered. In fact it is well-known that for noninteracting (or even repelling) particles ordering may be entropy driven. ${ }^{46-51}$ An example is provided by lyotropic phases that undergo disorder-order transitions upon increasing concentration, which in our case is equivalent to the surface density of Tc molecules. According to Onsager's model for a closed system of nonattracting rigid anisotropic objects (so-called "hard rods"), entropic ordering is driven by competing contributions of orientational and positional entropy. ${ }^{20}$ Repelling Tc molecules that are confined to the interface layer by the interaction with the substrate are a strictly 2D realization of Onsager's model. In this system the specific positional entropy (positional entropy per molecule) scales with the number of empty adsorption sites that are available to a molecule and hence decreases as the Tc coverage is increased. In contrast the specific orientational entropy depends only weakly on the coverage because it scales with the number of possible molecular orientations, and the latter are mainly defined by the substrate, at least for dilute Tc layers. Increasing the Tc coverage in the disordered $\delta$-phase, the molecular surface density will eventually reach the value of RCP, at which randomly oriented molecules lock each other in fixed positions. At this point the specific positional entropy approaches its minimum.

RCP densities are usually smaller than the densities achievable in ordered arrays of the same objects. ${ }^{52-54}$ Therefore, if the RCP layer of the $\delta$-phase orders, the positional entropy 
will increase because in the ordered structure each molecule gains some free area, which it can explore. In other words the positional locking of the RCP arrangement is lifted by the ordering (for this reason, positional entropy is sometimes referred to as "entropy of the free volume/area"). On the other hand ordering also leads to an abrupt decrease of the specific orientational entropy, as the orientation of all molecules will be now fixed, in case of the $\gamma$-phase by alignment with their neighbors. In entropically driven disorder-order transitions, as predicted by Onsager, the loss of orientational entropy is always overcompensated by the gain of positional entropy. The dominance of positional over orientational entropy in the present case of the $\delta \rightarrow \gamma$ transition at the $\mathrm{Tc} / \mathrm{Ag}(111)$ interface is strongly suggested by the influence of the substrate, which severely restricts the orientational freedom of Tc to three $\langle 01 \overline{1}\rangle$-directions of the substrate; hence, the specific orientational entropy is small from the outset and should play only a minor role. We therefore conclude that during early stages of ordering at the $\mathrm{Tc} / \mathrm{Ag}(111)$ interface domains of the $\gamma$-phase may appear as the result of a disorder-order phase transition at constant surface density that is driven by entropy.

For coverages larger than the one of the $\gamma$-phase, ordered ML structures with still higher surface densities are energetically favorable because additional molecules can be packed into the first layer, thus reducing the total energy of the molecular film. Placing additional molecules in the ordered $\gamma$ phase layer, however, requires a differently ordered interface. This is the driving force for the formation of the $\alpha$-phase (see Sec. IV A) that has a molecular surface density of $1 / 100 \AA^{2}$, as compared to $1 / 121 \AA^{2}$ for the $\gamma$-phase. Evidently, in forming the $\alpha$-phase, specific positional entropy that was gained in the $\delta \rightarrow \gamma$ transition is lost again because the free area of the $\gamma$-phase is occupied with additional molecules. Nevertheless, in spite of this entropy loss the $\alpha$-phase appears to be energetically favorable if compared to $\gamma$-phase plus second-layer molecules. The growth of $\alpha$-domains at larger surface coverages (cf. Sec. IIIE) is therefore energetically driven.

To further support our conjecture that the $\delta \rightarrow \gamma$ phase transition occurs at the coverage of RCP, we have determined the surface coverage of Tc molecules in a RCP layer on $\mathrm{Ag}(111)$ in a simulation, employing the random sequential adsorption (RSA) approximation. ${ }^{55}$ It assumes that molecules arrive at the surface sequentially in random positions and orientations (but always with the molecular plane parallel to the surface) and adsorb only if the chosen position is empty, i.e., if there is no steric hindrance with neighbors that are already on the surface. Otherwise the adsorption event is rejected. In this approximation position and/or orientation changes of the molecules after their adsorption are excluded ("hit and stick"). The last successfully placed molecule defines the total surface coverage from which the areal RCP density can be calculated. We note that this algorithm does not represent our actual adsorption experiments (i.e., deposition at high $T_{\text {dep }}$ and subsequent cooling, so diffusion and reorientation are possible), but it still gives a valid estimate of RCP density of $\mathrm{Tc}$ on $\mathrm{Ag}(111)$. Determining this density is the sole purpose of the simulations.

In detail the RSA simulation was carried out as follows. A hexagonal lattice $\left(r_{1}, r_{2}\right)$ with the lattice constant of $\operatorname{Ag}(111)$

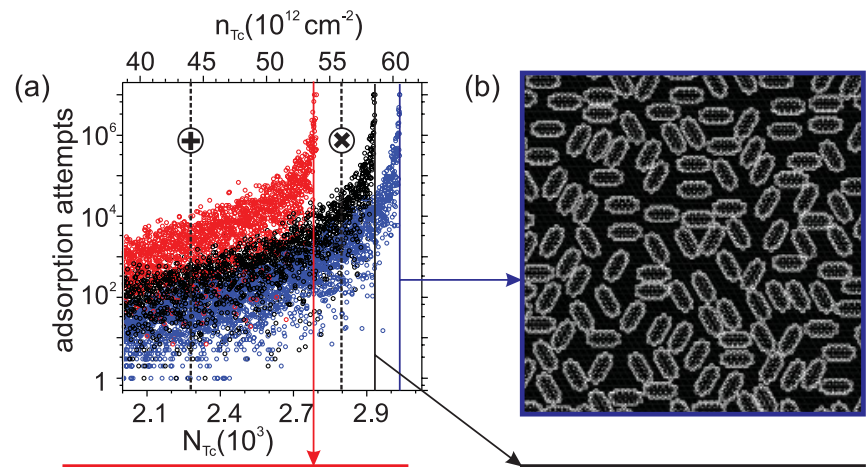

(c)

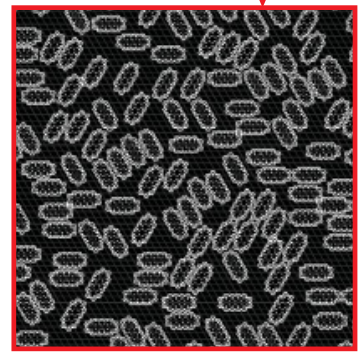

(d)

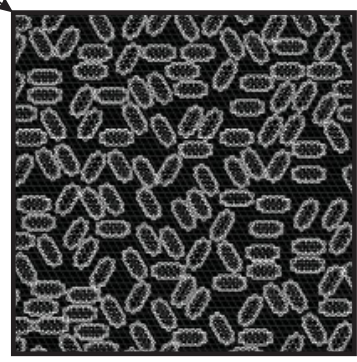

FIG. 4. (Color) Simulation of the random close-packed (RCP) Tc layer in the random sequential adsorption (RSA) approximation. (a) Number of single adsorption attempts needed for a successful event as function of the total number of Tc molecules $N_{\mathrm{Tc}}$ already placed on the surface (bottom axis) and the corresponding surface density of the layer $n_{\mathrm{Tc}}$ (top axis). Three cases are simulated-full registry with the $\operatorname{Ag}(111)$ substrate, i.e., commensurate structure (red dots); point-online registry with $\mathrm{Ag}(111)$, i.e., molecules placed on top of $\mathrm{Ag}$ atomic rows (black dots); and incommensurate adsorption (blue dots). The results of three independent simulations are accumulated for each case in the plot. The surface density at which the number of required attempts diverges was taken as the density of the corresponding RCP phase (red, black, and blue vertical lines). Black dashed lines and symbols $\boldsymbol{+}, \boldsymbol{x}$ represent the surface densities in the STM images of Figs. 3(a) and 3(b). (b)-(d) Distribution of Tc molecules from the RCP simulations in cases of incommensurate registry (b), commensurate registry (c), and point-on-line registry (d).

$\left(\left|r_{1}\right|=\left|r_{2}\right|=2.9 \AA\right)$ was defined. To be able to model incommensurate registries of Tc on the silver substrate as well as commensurate ones, a smaller (also hexagonal) mesh was defined with $t_{1}=1 / 6 r_{1}$ and $t_{2}=1 / 6 r_{2}$. Thus, every sixth position in the hexagonal direction represents the position of a silver atom, with five possible adsorption positions in between any two silver atoms. For the modeling of incommensurate adsorption, any of the lattice points $\left(t_{1}, t_{2}\right)$ was allowed, while for the modeling of commensurate adsorption, molecules were placed on the $\left(r_{1}, r_{2}\right)$ lattice only. The orientation of Tc molecules was restricted to three hexagonal directions of the $\operatorname{Ag}(111)$ lattice. New molecules were placed until the next molecule could not be placed in $10^{7}$ iterative attempts. The asymptotic behavior in Fig. 4(a) shows that this threshold is a reasonable approximation of the RCP surface density. Finally, averaging the results of ten simulations for each case of registry, the maximal possible surface density was calculated. The footprint of the molecule was taken from Ref. 1.

The thus determined RCP density of Tc molecules on $\operatorname{Ag}(111)$ is $5.4 \times 10^{13} \mathrm{~cm}^{-2}$ for adsorption at silverlattice sites only (corresponding to commensurate registry), 
$5.9 \times 10^{13} \mathrm{~cm}^{-2}$ for adsorption on the $t$-lattice on silver-lattice lines only (mimicking point-on-line registry), and $6.1 \times 10^{13}$ $\mathrm{cm}^{-2}$ for adsorption at arbitrary $t$-sites (mimicking incommensurate registry). All three simulated RCP densities are close to experimental observation: The Tc/Ag(111) interface is disordered at $4.4 \times 10^{13} \mathrm{~cm}^{-2}$ coverage [Fig. 3(a)], while first signs of ordering are registered at the coverage of $5.6 \times$ $10^{13} \mathrm{~cm}^{-2}$ [Fig. 3(b)]. However, only one of the simulations, namely the case of commensurability with the substrate, yields a maximum coverage that is between the disordered arrangement [Fig. 3(a) and + symbol in Fig. 4(a)] and a partially $\gamma$-ordered arrangement [Fig. 3(b) and $\boldsymbol{*}$ symbol in Fig. 4(a)]. It thus seems as if in our experiments the ordering into the $\gamma$-phase sets in close to commensurate RCP coverage of $\mathrm{Tc}$ on $\mathrm{Ag}(111)$, as previously conjectured. This again suggests that the $\gamma$-phase is commensurate. Indeed, the unit cell observed in $\operatorname{STM}\left(\left|\mathbf{g}_{1}\right| \approx\left|\mathbf{g}_{2}\right| \approx 13 \AA, \gamma \approx 135^{\circ}\right)$ is very close to the geometrically constructed commensurate unit cell $\left(\left|\mathbf{g}_{1}\right|=\left|\mathbf{g}_{2}\right|=12.60 \AA, \gamma=133.14^{\circ}\right)$ at $\operatorname{Ag}(111)$.

We note here that our RSA simulation does not take into account intermolecular repulsion. Comparing the pattern in Figs. 3(a)-3(c) with the one in Fig. 4(c), one notices that in the experiment side-by-side arrangements are less frequent than in the simulation. In the experiment various local head-to-head configurations with molecules rotated with respect to each other seem to be more prevalent. This comparison between experiment and simulation confirms that there is a repulsive interaction between Tc molecules that is particularly strong if these are orientated side-by-side. This suggests an anisotropic repulsion between Tc molecules that is weaker in the head-totail than in the side-by-side configuration.

We have also simulated the adsorption process, including the possibility of surface diffusion along the close-packed directions of $\operatorname{Ag}(111)$ and rotation at the high symmetry positions. In these simulations the threshold of $10^{7}$ iterations for a successful adsorption event was reached at much higher surface densities (approximately $8.0 \times 10^{13} \mathrm{~cm}^{-2}$ ). However, a tendency for side-by-side alignments of Tc molecules (and finally clustering of parallel molecule) is observed simultaneously in extended areas, which is not found in our STM images. We note that this does not imply that in our experiments diffusion and rotation are impossible; rather, the intermolecular repulsion, which is missing from the simulation but present in reality, suppresses the parallel orientation in experiment. Therefore, the RSA without diffusion and rotation is closer to reality because it does not favor side-by-side alignments, as the RSA with diffusion and orientation apparently does. It is not surprising that our strictly steric simulation does not provide a proper description for the growth process, because all interaction potentials and kinetic coefficients for the surface diffusion and rotation are ignored. However, this oversimplified approach seems to predict characteristic densities correctly (see the previous discussion).

\section{E. $\alpha$-phase}

In the case of room temperature deposition $(300 \mathrm{~K})$ and subsequent cooling to below $180 \mathrm{~K}$, the long-range ordered $\alpha$-phase appears. In Figs. 5(a) and 5(c) the diffraction pattern and a real-space STM image of the $\alpha$-phase are displayed. The
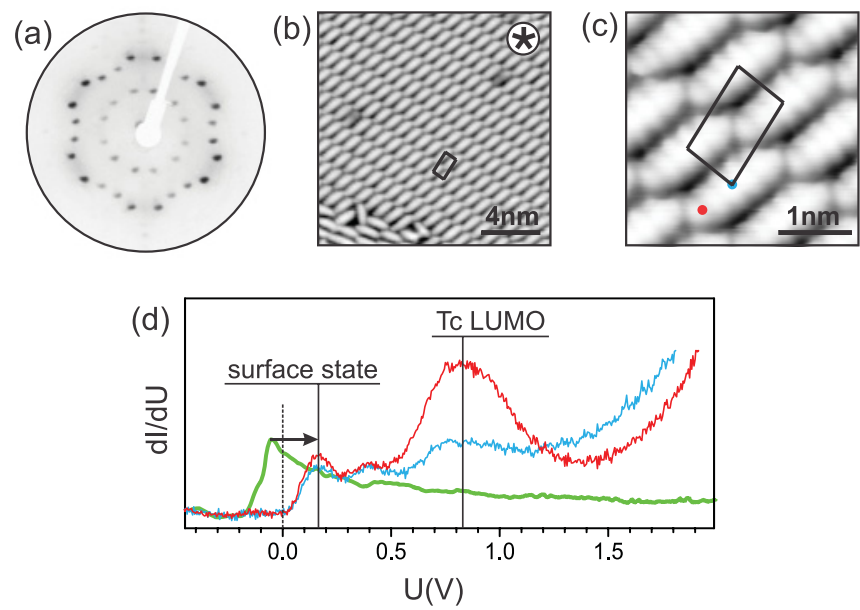

(e)

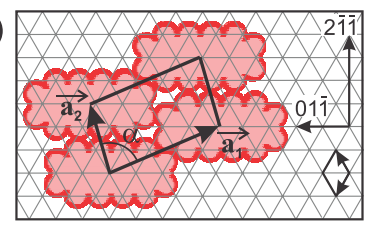

FIG. 5. (Color) (a) LEED pattern (beam energy $12 \mathrm{eV}$ ) and (b), (c) STM images (tunneling current $0.1 \mathrm{nA}$, bias voltage $1.5 \mathrm{~V}$ ) of the $\mathrm{Tc} / \operatorname{Ag}(111) \alpha$-phase. The unit cell is marked in (b) and (c). (d) $\mathrm{dI} / \mathrm{dV}$ spectra of a Tc molecule in the $\alpha$-phase, recorded at its center [blue curve, approximate position marked in blue in (c)] and its edge [red curve, approximate position marked in red in (c)]. The green curve is the spectrum of the clean $\operatorname{Ag}(111)$ surface. The black horizontal arrow marks the shift of the $\mathrm{Ag}(111)$ surface state upon Tc adsorption. (e) Unit cell of the $\alpha$-phase (angle and unit cell vectors are taken from SPA-LEED data of Langner et al. ${ }^{1}: \mathbf{a}_{1}=12.9 \AA, \mathbf{a}_{2}=7.8 \AA, \alpha=$ $\left.83.2^{\circ}\right)$.

STM micrographs reveal a close-packed layer of molecules, similar to the structure model suggested in Ref. 1 . The feature size $(14 \AA \times 7 \AA)$ suggests flat-lying molecules. Again, the direction of molecules coincides with $\langle 01 \overline{1}\rangle$-directions of the $\operatorname{Ag}(111)$ crystal surface, and the unit cell determined from $\operatorname{STM}\left(a_{1}=8.2 \AA, a_{2}=13.1 \AA, \alpha=93^{\circ}\right)$ is close to the one derived from electron diffraction $\left(a_{1}=7.8 \AA, a_{2}=12.9 \AA\right.$, $\left.\alpha=96.8^{\circ}\right){ }^{1}$ Consequently, our results confirm the previous findings about the structure of the $\alpha$-phase. The $\alpha$-phase exhibits a point-on-line registry ${ }^{1}$ [Fig. 5(e)].

In differential conductance spectra $(\mathrm{dI} / \mathrm{dV})$ recorded above a single Tc molecule in the $\alpha$-phase [Fig. 5(d), black curve], a broad resonance at $+0.8 \mathrm{eV}$ is observed. It is stronger at the edges of Tc molecules and almost vanishes close to the center [corresponding blue and red dots in Fig. 5(c)]. Scanning at this energy with a Tc functionalized tip ${ }^{38,56,57}$ clearly reveals the characteristic geometry of the LUMO, as calculated ${ }^{58}$ for the free Tc molecule-four narrow lobes that are oriented perpendicular to the long molecular axis in the center of the molecule and two more circular and bright features at either end of the molecule. The submolecularly resolved image of the molecules confirms that in the $\alpha$-phase molecules indeed adsorb with their molecular plane parallel to the substrate. Scanning with a pure metal tip resolves the LUMO geometry less clearly. 
In the $\alpha$-phase the originally filled surface state of $\operatorname{Ag}(111)$ $\left(-67 \mathrm{meV}^{59-61}\right)$ is depopulated and appears in tunneling spectra upshifted by about $220 \mathrm{meV}$ [Fig. 5(d)]. A noticeable peak at $+0.4 \mathrm{eV}$ is due to a scattering of electron waves of silver surface state at the silver step edge. This upshift of the Shockley surface state by an amount of this order is well-known from the physisorption of noble gases on transition metal (111)-surfaces. ${ }^{45}$ In the case of noble gases the origin is the Pauli repulsion between the closed shell of the adsorbate and the electrons in the surface state, which reaches out relatively far into the vacuum. This repulsion leads to a slight destabilization of the adsorbate. In the present case we can estimate the destabilization energy as $8 \mathrm{meV}$ per molecule (cf. Sec. III D), which compares, e.g., to $50 \mathrm{meV}$ per Xe atom for adsorption in the $\sqrt{3} \times \sqrt{3} \mathrm{R} 30^{\circ}$ phase on $\operatorname{Pt}(111){ }^{45}$ We note here that for $\mathrm{Xe} / \mathrm{Pt}(111)$, the depopulation of the surface state also causes an attractive interaction between neighboring $\mathrm{Xe}$ adsorbates, leading to island growth. As mentioned previously, in the present case of $\mathrm{Tc} / \operatorname{Ag}(111)$ we do not observe island growth of the $\alpha$-phase (or $\gamma$-phase, for that matter) from the lattice gas. This indicates that the repulsive interaction between $\mathrm{Tc}$ molecules on $\operatorname{Ag}(111)$ is stronger than any surface-state mediated attraction that may exist here. A possible origin of this repulsion is the dipole-dipole interaction between parallel adsorption dipoles.

If $T_{\text {dep }} \leqslant 100 \mathrm{~K}$, the $\alpha$-phase is not observed after cooling. The interface remains disordered, although with STM we observed substantial surface mobility of Tc even at much lower temperature (cf. previous section). Here the peculiar behavior of the $\mathrm{Tc} / \operatorname{Ag}(111)$ interface with its intermolecular repulsion offers a possible explanation. Due to intermolecular repulsion, ordered phases can only nucleate from a homogeneous disordered phase once the coverage exceeds RCP. No nucleation from a $2 \mathrm{D}$ gas phase is possible. This means that for deposition at $T_{\text {dep }} \leqslant 100 \mathrm{~K}$ the $\alpha$-phase can only form once the $\delta$ - and $\gamma$-phases have been traversed (cf. Fig. 1). In this context it is noteworthy that the structural motifs of the $\gamma$-phase and the $\alpha$-phase are very similar [Figs. 6(a) and 6(b)]. For example, they both contain pairs of Tc molecules that are arranged corner-to-corner, and, moreover, the unit cell vectors that correspond to this translation are also very close to each other. They have lengths of approximately $13 \AA$ (12.6 $\AA$ for ideal commensurate) and $13.1 \AA$ (12.9 $\AA$ from SPA-LEED ${ }^{1}$ ), respectively, for $\gamma$ and $\alpha$ (cf. Table I). Accordingly, the structural transformation from the less dense but commensurate $\gamma$-phase into the more dense but only point-on-line coincident $\alpha$-phase requires a lateral shift of one of the molecular chains in Figs. 6(a) and 6(b), as shown schematically in Fig. 6(c) (yellow arrow). Due to steric hindrance the transition from the $\gamma$-phase to the $\alpha$-phase may involve the necessity to temporarily escape into third dimension (or second layer), which, due to energetic reasons, is only possible at elevated temperatures.

\section{F. Force-field calculations for the $\boldsymbol{\gamma}$ - and $\alpha$-phases}

In the previous sections we have discussed the general driving forces that favor structural order at the tetracene $/ \operatorname{Ag}(111)$ interface. We now want to address the question why in particular the $\gamma$ - and $\alpha$-phases form with their (a)

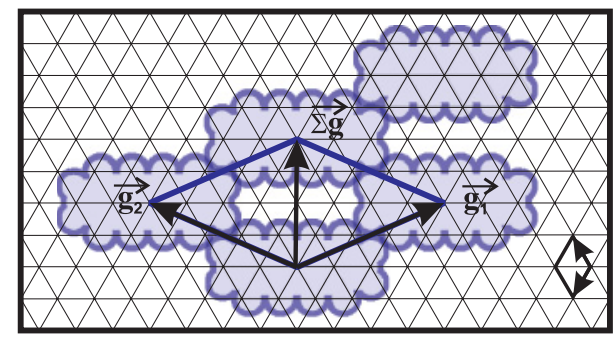

(b)

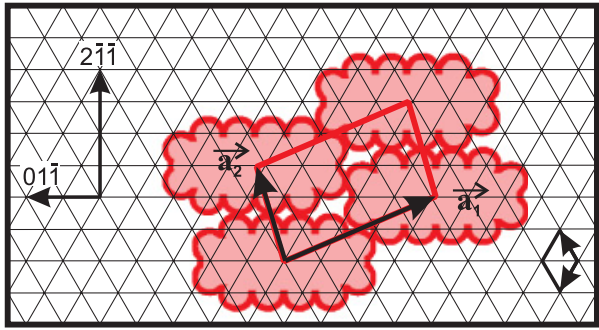

(c)

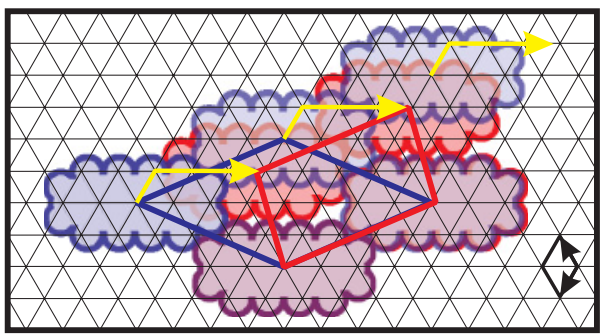

FIG. 6. (Color) Unit cells of the (a) $\gamma$-phase and (b) $\alpha$-phase of $\mathrm{Tc} / \operatorname{Ag}(111)$. The $\gamma$-phase is plotted as a commensurate superstructure. The $\alpha$-phase is plotted according to SPA-LEED data from Ref. 1. (c) The $\gamma$-phase can be transformed into the $\alpha$-phase by a rigid displacement of Tc chains as indicated with the yellow arrows.

respective unit cells and molecular packing. To this purpose, we employ force-field calculations. While these calculations are no substitute for detailed ab initio simulations, they do provide first hints that allow the rationalization of structural motifs in molecular films. ${ }^{9,19,62}$

Our force-field calculations explicitly take into account molecule-molecule interactions only. The interaction with the substrate is taken into consideration only insofar as in our calculations we restrict the orientations and positions of the molecules relative to the substrate lattice to those which are observed in experiment. As previously discussed in Secs. III C-IIIE, experimental data show that Tc molecules in the $\gamma$ - and $\alpha$-phases are oriented with their long axes parallel to the $\langle 01 \overline{1}\rangle$ high-symmetry directions; moreover, their nearest-neighbor distances perpendicular to this direction very nearly coincide with the distance between four atomic rows of the substrate. It is therefore indicated by experiment that in the $\gamma$-and $\alpha$-phases all Tc molecules are located on substrate lattice lines that run parallel to their long axes. This is assumed in the force field calculations to be subsequently discussed. Apart from this, no interaction with the substrate is included into the calculation.

Two contributions to the intermolecular interaction are considered, the van der Waals interaction and the electrostatic interaction. To calculate these, the coordinates of the atomic nuclei are extracted from the undistorted gas phase geometry of Tc. The van der Waals interaction energy is calculated 
by summation over all atom pairs in neighboring molecules using the parameters of Refs. 63-66 for the repulsive (Pauli) and the attractive (London) contributions. For the calculation of the electrostatic interaction, as a first step partial charges of all nuclei in the Tc molecule are determined, employing the natural-bond orbitals-population analysis ${ }^{62}$ based on DFT calculations for the free molecule. ${ }^{58}$ The total electrostaticinteraction energy is then calculated by summation over all atom pairs in neighboring molecules. We note in particular that a possible charge transfer between the metal and adsorbed Tc molecules is not accounted for. Details of the calculation method can be found elsewhere. ${ }^{62}$

In Fig. 7(a) a potential map for a pair of coplanar, parallel Tc molecules is displayed. One of the molecules is located in the center of the graph; the other is moved from point-to-point in the $x y$-plane. For each $x y$-position, the total interaction energy is calculated. The color code represents the calculated potential energy of the Tc pair. Positions inside the black region in the center of Fig. 7(a) are excluded for steric reasons. The zero-point of the potential energy scale corresponds to the case of two molecules at infinite distance from each other.

We observe that for most relative positions the interaction between the two Tc molecules is repulsive. This is consistent with our experimental finding that at low surface densities, Tc molecules spread out evenly on the surface and do not nucleate into islands. However, at relatively close distance we find 12 minima, in which the potential energy becomes negative, i.e., attractive (note that only three of the twelve minima are nonequivalent by symmetry). The area around each minimum in which the interaction is attractive is very small (approximately $1 \AA^{2}$ ). Remarkably, four of the twelve minima are located on lattice lines of the substrate. They are labeled with the vectors $\mathbf{g}_{1}{ }^{\prime}, \mathbf{g}_{2}{ }^{\prime},-\mathbf{g}_{1}{ }^{\prime}$, and $-\mathbf{g}_{2}{ }^{\prime}$ in Fig. 7(a). If Tc molecules sit in these sites, they can simultaneously minimize their interaction with the substrate (via point-on-line registry) and with the Tc molecule at the center. Interestingly, the angles $\angle\left(\mathbf{g}_{1}{ }^{\prime}, \mathbf{g}_{2}{ }^{\prime}\right)$ and $\angle\left(-\mathbf{g}_{1}{ }^{\prime},-\mathbf{g}_{2}{ }^{\prime}\right)$ are $134.4^{\circ}$, which is very close to the angle between unit cell vectors of $\gamma$ phase, as derived from STM $\left(135^{\circ}\right)$ or suggested by assumed commensurate arrangement $\left(133.14^{\circ}\right.$ ) (cf. Sec. III C, III D, and Table I). Moreover, the length of the vectors $\mathbf{g}_{1}{ }^{\prime}, \mathbf{g}_{2}{ }^{\prime},-\mathbf{g}_{1}{ }^{\prime}$, and $-\mathbf{g}_{2}{ }^{\prime}(12.9 \AA)$ almost perfectly agrees with corresponding unit cell vector of $\gamma$-phase $(13 \AA$ as derived from STM or $12.60 \AA$ of commensurate structure). We can thus conclude that $\mathbf{g}_{1}{ }^{\prime} \approx$ $\mathbf{g}_{1}$ and $\mathbf{g}_{2}{ }^{\prime} \approx \mathbf{g}_{2}$, and hence the structural motif of the $\gamma$-phase, as shown in Fig. 3(d), follows naturally if the Tc molecules in an ordered layer are to minimize both their interaction with the substrate and with their neighbors.

We now turn to the $\alpha$-phase. Figure 7(a) shows that the intermolecular interaction energy in the $\alpha$-phase with its unit vectors $\mathbf{a}_{1}$ and $\mathbf{a}_{2}$ is larger because, in particular, the vector $\mathbf{a}_{2}$ is not located close to a local minimum in the potential energy landscape of a pair of Tc molecules. What, then, determines the unit cell for the $\alpha$-phase? In Fig. 6 we have seen that the $\alpha$-phase can be generated from the $\gamma$-phase by rigidly translating a molecular row. In Fig. 7(b) we have plotted the potential energy landscape for the interaction of two parallel rows of Tc molecules. In this plot the structure of the $\gamma$-phase, represented by the vectors $\mathbf{g}_{1}{ }^{\prime}, \mathbf{g}_{2}{ }^{\prime}$ and $\Sigma \mathbf{g}^{\prime}$, again corresponds to a minimum in intermolecular interaction energy. In contrast
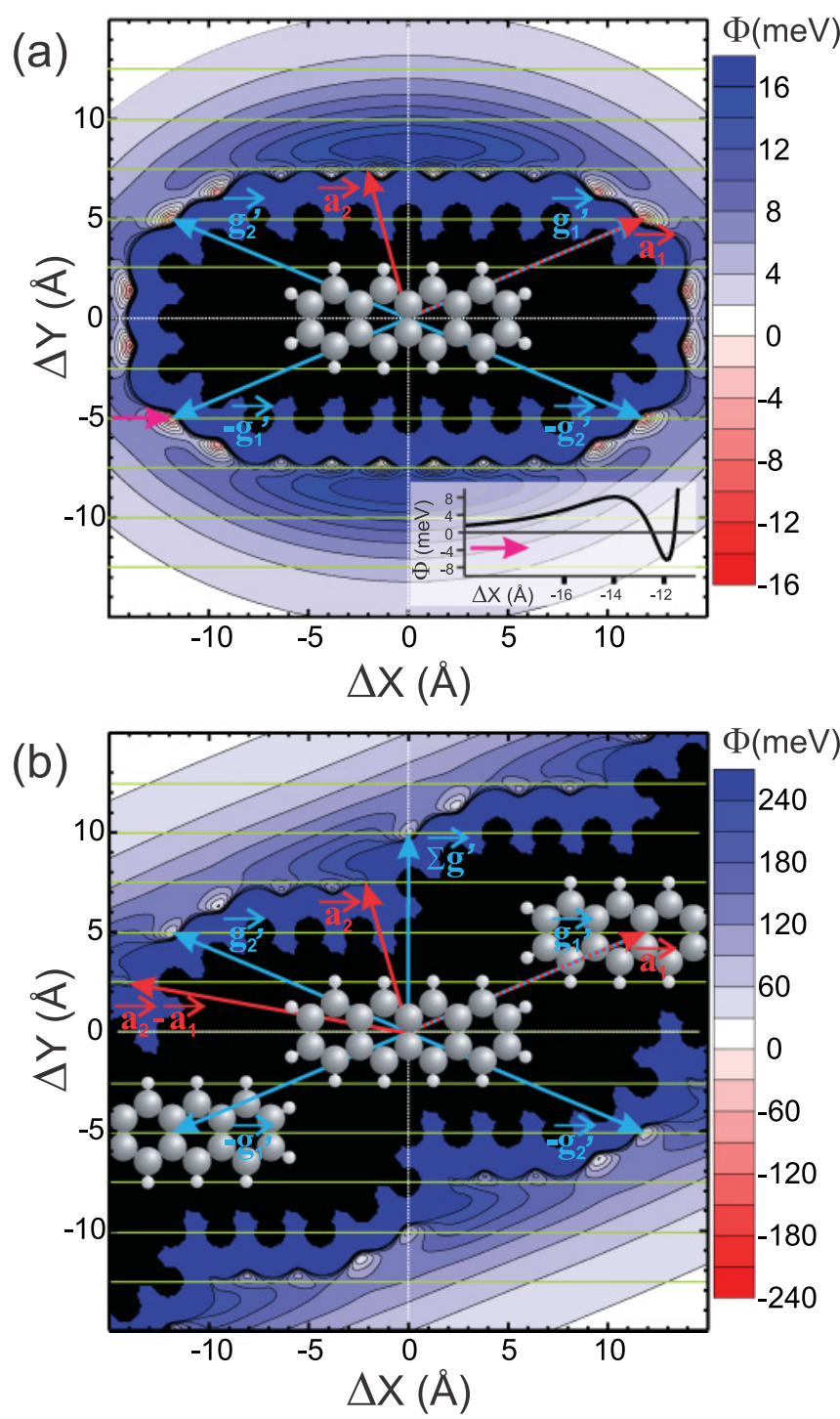

FIG. 7. (Color) (a) Intermolecular interaction energy $\Phi$ as a function of relative position of two co-planar Tc molecules that are aligned parallel. One molecule (shown) is located at the center of the graph, the other (not shown) is displaced by $\Delta \mathrm{X}, \Delta \mathrm{Y}$ relative to it. (b) Intermolecular interaction energy $\Phi$ as a function of relative position of two co-planar, parallel chains of Tc molecules (nine molecules in each chain). One chain (shown) is located at the center of the graph, the other (not shown) is rigidly displaced by $\Delta \mathrm{X}, \Delta \mathrm{Y}$ relative to it. The black region in the $(\Delta \mathrm{X}, \Delta \mathrm{Y})$ map is excluded because of steric hindrance. The vectors $\mathbf{g}_{1}{ }^{\prime}, \mathbf{g}_{2}{ }^{\prime},-\mathbf{g}_{1}{ }^{\prime},-\mathbf{g}_{2}{ }^{\prime}$, and $\Sigma \mathbf{g}^{\prime}$ point to the local potential minima which agree with unit cell vectors of the $\boldsymbol{\gamma}$-phase. Vectors $\mathbf{a}_{1}$ and $\mathbf{a}_{2}$ are the unit cell vectors of $\boldsymbol{\alpha}$-phase. Inset in (a) shows the potential profile for a Tc molecule diffusing along a silver atomic row (purple arrow) and approaching another Tc molecule fixed in the center of the map.

we see that the vectors of the $\alpha$-phase are determined by the closest possible packing of the chains, i.e., the two vectors $\mathbf{a}_{2}$ and $\mathbf{a}_{2}-\mathbf{a}_{1}$ are the shortest possible unit cell vectors on the border of the region where repulsion rises very steeply (cf. the isopotential lines in Fig. 7(b)). We can thus conclude that apart from the necessary avoidance of steric hindrance, the intermolecular interaction does not play a role in determining 
the $\alpha$-phase structure. Rather, it is the attractive moleculesubstrate interaction that determines the structure of the $\alpha$ phase, because in this structure maximal packing density can be achieved.

\section{IV. $\beta$-PHASE $(\theta>1$ ML $)$}

In the case of low temperature deposition $\left[T_{\text {dep }} \leqslant 230 \mathrm{~K}\right.$, cf. Fig. 1(b)] and subsequent cooling below $180 \mathrm{~K}$ another type of a long-range ordered superstructure appears on $\operatorname{Ag}(111)$ if the total exposure is approximately $2.23 \mathrm{ML}$ - the $\beta$-phase. ${ }^{1}$ According to Ref. 1 , the $\beta$-phase is characterized by two different periodicities, a fundamental unit cell with dimensions $\left|\mathbf{b}_{1}\right|=10.6 \AA$ and $\left|\mathbf{b}_{2}\right|=13.9 \AA$, which includes two tilted Tc molecules, and a periodic supercell including domain walls. As we will see, this is partially confirmed by the present STM data. Because of the larger coverage that leaves less footprint area for each molecule, it was suggested in Ref. 1 that in the $\beta$-phase molecules tilt out of the surface plane. This was an unexpected result because $\pi$-conjugated molecules usually adsorb on metals with their plane parallel to the surface in order to minimize their energy. However, we will show below the detailed molecular structure is more complicated than suggested in Ref. 1. In particular, the inclination of first layer molecules out of the interface plane is induced by their interaction with Tc molecules in the second layer.

In the STM micrograph shown in Fig. 8 , the $\beta$-phase appears higher by $2.7 \AA$ than the $\alpha$-phase domain on the

(a)

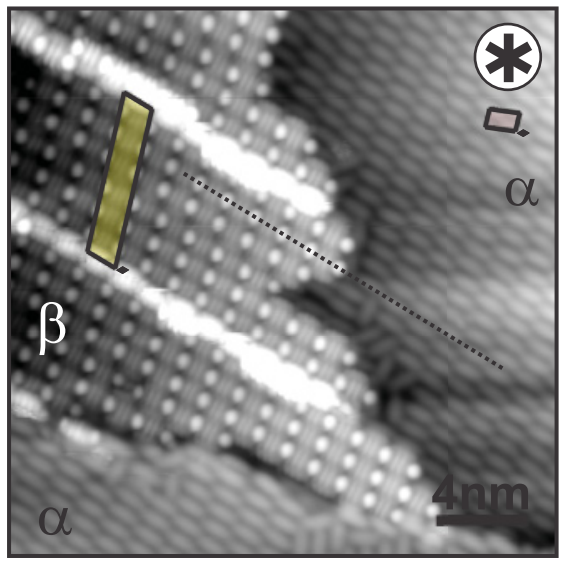

(b)

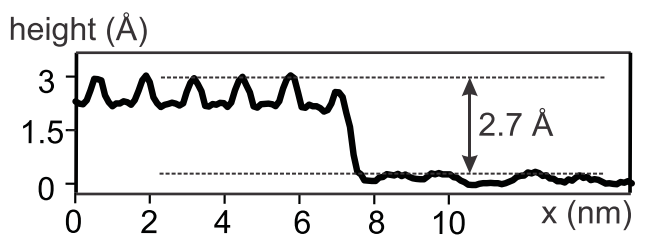

FIG. 8. (Color) (a) STM image (bias voltage $2 \mathrm{~V}$, tunneling current $0.7 \mathrm{nA}$ ) of $\mathrm{Tc} / \mathrm{Ag}(111)$ showing $\alpha$ - and $\beta$-phases of Tc coexisting on the same terrace of the substrate. The $*$ symbol indicates the point in the phase diagram of Fig. 1 that corresponds to the STM image. The unit cells of the $\alpha$ - and $\beta$-phases are marked in black and shaded in yellow or red, respectively. For comparison, the $\operatorname{Ag}(111)$ unit cell is shown by small black rhombus. (b) STM height profile recorded along the dashed line in (a). same Ag terrace [in comparison, a step from the $\operatorname{Ag}(111)$ to the $a$-phase is $1.4 \AA]$. This suggests that the $\beta$-phase is a bilayer of Tc (although due to electronic structure effects, the latter numbers cannot be taken as an indicator of the actual height difference between $\alpha$ - and $\beta$-phases). In particular one observes a compact arrangement of molecules in what appears to be the second layer plus chains in the third layer. Although at this point the designation of second and third layer is ad hoc, the structural analysis unambiguously confirms this assignment. Also, it is apparent that the $\alpha$-phase is disrupted at the borderline of the $\beta$-phase, suggesting that the latter is not just a second layer on top of the unmodified $\alpha$-phase.

\section{A. Preparation of the $\beta$-phase}

The $\beta$-phase can only be prepared under special circumstances. (1) There must be enough material present to form the $\beta$-phase, i.e., the average coverage of the surface with Tc molecules must exceed the surface density of $1.0 \times$ $10^{14}$ molecules $/ \mathrm{cm}^{2}$, which corresponds to the $\alpha$-phase. (2) During or after deposition of Tc, the surface must never have been exposed to temperatures larger than $230 \mathrm{~K}$. If these conditions are fulfilled at least part of the $\operatorname{Ag}(111)$ surface will be covered by the $\beta$-phase. If, e.g., the coverage is intermediate-more than $1.0 \times 10^{14}$ molecules $/ \mathrm{cm}^{2}(\alpha$-phase surface density) but less than $2.23 \times 10^{14}$ molecules $/ \mathrm{cm}^{2}$ ( $\beta$-phase surface density) — and if the temperature has always stayed below $230 \mathrm{~K}$, both the $\alpha$ - and the $\beta$-phases coexist at the surface (Fig. 8). If, on the other hand, more than $2.23 \times$ $10^{14}$ molecules $/ \mathrm{cm}^{2}$ is supplied and again the temperature is always kept below $230 \mathrm{~K}$, the $\beta$-phase forms everywhere on the sample, and the excess molecules end up in 3D clusters. ${ }^{1}$ Note that if this same amount is supplied above $230 \mathrm{~K}$, the sample will be covered completely with $\alpha$-phase, and no $\beta$-phase is formed; surplus material is then either lost from the surface via desorption or taken up in clusters. Even if during the cooling process the temperature of $230 \mathrm{~K}$, at which, if deposited there, the $\beta$-phase would form, is passed, and enough material is present for the $\beta$-phase, it does not form. Incidentally, the $\beta$-phase is also metastable in the sense that it does not survive annealing above $230 \mathrm{~K}$ (see Sec. IV D). We finally note that the nature of the $\alpha$-phase is principally different from that of the $\beta$-phase because, first, it survives annealing up to the point when Tc is desorbed from the surface, and second, it forms in a given coverage range irrespective of the temperature at which molecules are deposited (except for $T_{\text {dep }} \leqslant 100 \mathrm{~K}$, see Sec. III E).

\section{B. Structural organization of the $\beta$-phase: Interface layer, surface layer, domain walls}

It turns out that despite the complexity of the STM image in Fig. 8, the structural organization of the $\beta$-phase can be derived on the basis of STM images. For this the possibility to image the $\beta$-phase in two layers turns out to be crucial. If voltages above $1.8 \mathrm{~V}$ are used, one records images [Fig. 9(a)] like the one shown in Fig. 8. On the other hand for bias voltages below $1.8 \mathrm{~V}$ the images change drastically, as can be seen in Figs. 9(b) and 9(c). Notably, the areal density of features in these images [compare Fig. 9(a)-9(c)] is different. 
(a)
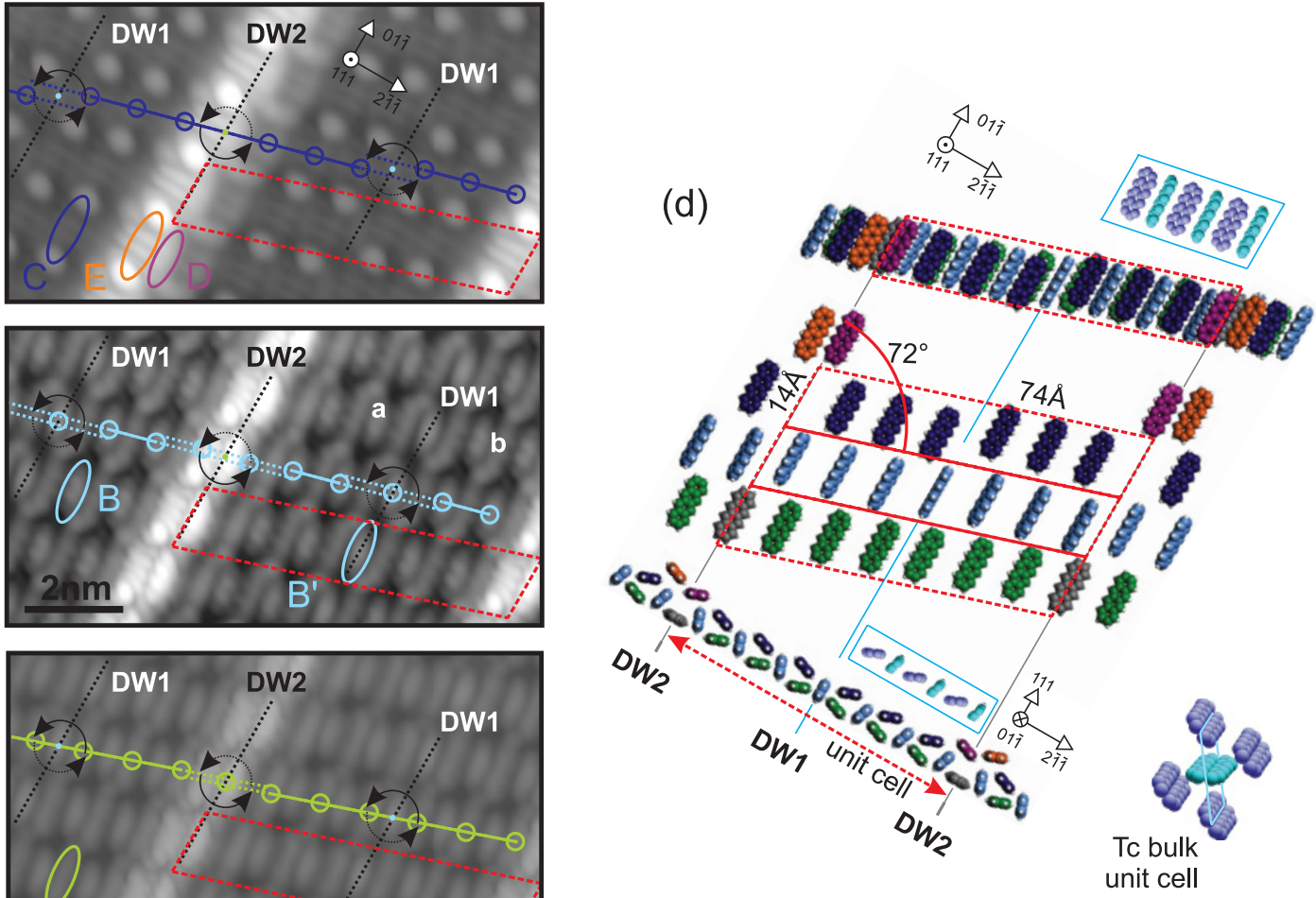

(b)

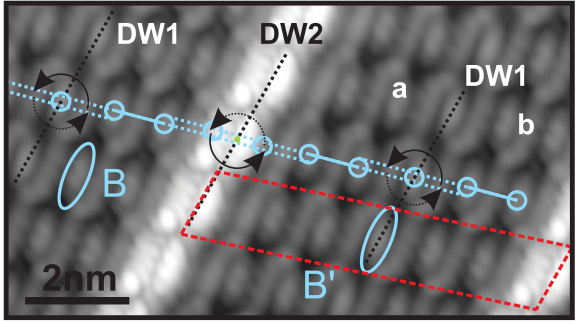

(c)

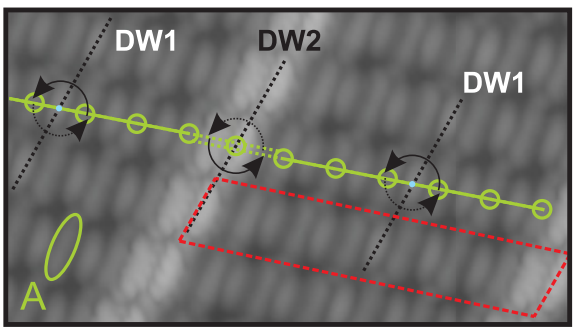

unit cell

FIG. 9. (Color) (a)-(c) STM images of the Tc/Ag(111) $\beta$-phase acquired at different bias voltages and tunneling currents: (a) $2.0 \mathrm{~V}$, $47 \mathrm{pA}$; (b) $1.0 \mathrm{~V}, 47 \mathrm{pA}$; (c) $0.6 \mathrm{~V}, 47 \mathrm{pA}$. Molecules in different sublattices are marked with colored ovals and letters A, B (B'), C, D, E; The center positions of molecules in the A, B, and C sublattices are marked with green, light blue, and dark blue circles in (a), (b), and (c), respectively. The positions of domain walls are marked with black dotted lines that are labeled DW1 or DW2. Red dotted lines show the unit cell of the $\beta$-phase. Black circular arrows mark the positions of two-fold rotation axes $\mathrm{R}_{2}$ (see main text). (d) Structure model for the Tc/Ag(111) $\beta$-phase: top view (top) and side view (bottom) of the unit cell (red dashed lines) with 22 molecules, color-coded as in panels (a)-(c). Between the top and side views the various sublattices are shown separately. Positions of DW1 and DW2 are indicated. For comparison with the $\beta$-phase, the molecules in the blue boxes show the structure motif of bulk Tc. Inset: Unit cell of bulk Tc.

It therefore appears that different layers within the $\beta$-phase are imaged with the two sets of scanning parameters. A careful analysis of the electronic spectra, recorded with STS, confirms this conjecture, as the discussion in Ref. 35 shows. In short because of strong shifts in the energy position of the LUMO level due to site-specific polarization screening, it is possible at bias voltages below $1.8 \mathrm{~V}$ to image the lower (i.e., interface) layer of the $\beta$-phase through the band gap of the molecules in the upper (i.e., surface) layer, while above 1.8. V the surface layer is imaged. This imaging of the $\beta$-phase in slices relies on the effect that polarization screening by the image charge in the metal surface moves the molecular LUMO level, that is, the closer to the Fermi energy the closer the molecule is to the interface with the metal. Before discussing the molecular arrangements in both layers, i.e., the interface layer in Fig. 9(c) and surface layer in Fig. 9(a), we turn to another notable structural feature that is visible in the STM images.

In Figs. 8 and 9(a)-9(c) we observe two equidistant features with a long-range periodicity along the $\langle 2 \overline{1} \overline{1}\rangle$-direction: first domain walls (marked by DW1 in Fig. 9) and second chains in the third layer (marked by DW2 in Fig. 9). Both features have the same periodicity, but they are phase shifted with respect to each other such that domain walls (DW1) and chains (DW2) always appear alternatingly. The domain walls DW1 are shifts between adjacent domains along the long molecular axis, i.e., along the close-packed direction of the crystal face. The wavelength observed in Fig. 9 agrees very well with domain structure inferred from Ref. 1 .

We now turn to the analysis of the molecular structure of the $\beta$-phase. To this purpose, we have in Fig. 9 plotted high-resolution zoom images of the $\beta$-phase recorded at three different bias voltages. Figures 9 (b) and 9 (c) have been recorded below $1.8 \mathrm{~V}$, and therefore must be assigned to the interface layer. Indeed, there is a one-to-one correspondence between the features in Figs. 9(b) and 9(c), confirming that in both images the same molecular structure is imaged, albeit with slightly different contrasts. Generally speaking, Fig. 9(b) shows slightly better resolution. Figure 9(a), recorded with $2.0 \mathrm{~V}$, pertains to the surface layer. Note that due to topographic effects, the third-layer chains are always visible, even in images of the interfacial layer. For this reason we restrict our discussion of the interfacial layer in Figs. 9(b) and 9(c) on the area in between the chains.

We start with the surface layer shown in Fig. 9(a). This layer consists of only one type of molecule. It is marked with $\mathrm{C}$ (here and later the notation is the same as in Ref. 35). Its contrast is similar to the submolecular structure of molecules in the $\alpha$-phase, i.e., four narrow lobes that are oriented perpendicular 
to the long molecular axis in the center of the molecule and two more circular features at either end of the molecule. This suggests a similar orientation with respect to the surface, i.e., we can conclude that the plane of the $\mathrm{C}$ molecule is nearly parallel to the surface. Note that the contrast of the $\mathrm{C}$ molecules in the surface layer of the $\beta$-phase is higher than that of the molecules in the $\alpha$-phase, which follows naturally from the fact that the $\mathrm{C}$ molecule is not in contact with the metal (concordantly, its LUMO appears at $1.84 \mathrm{eV}$ instead of $0.8 \mathrm{eV}$ for the $\alpha$-molecule). Also note that under the experimental conditions of Fig. 9(a), the very bright lobes at either end of a $\mathrm{C}$ molecule form a joint protrusion with their direct neighbors along the $\langle 01 \overline{1}\rangle$-direction. Finally, in Fig. 9(a) the center positions of $\mathrm{C}$ molecules in adjacent rows are indicated by open circles. The translation of these circles by $10.9 \AA$ in the direction of $\mathrm{C}$ molecule periodicity (solid blue lines, rotated by $76^{\circ}$ degrees from $\langle 01 \overline{1}\rangle$ toward $\left.\langle 2 \overline{1} \overline{1}\rangle\right)$ is close to the fundamental unit cell vector of the $\beta$-phase (10.6 $\AA$ ) predicted by SPA-LEED. ${ }^{1}$ Looking at the molecule positions across the domain wall DW1, one clearly sees the axial shift of adjacent $\mathrm{C}$ molecules along the direction of the molecular rows $(\langle 01 \overline{1}\rangle)$ by approximately $3 \AA$.

The interface layer consists of several species of different shapes that form parallel rows along the $\langle 01 \overline{1}\rangle$-direction. (1) An elongated feature marked with $\mathrm{A}$ in Fig. 9(c) (LUMO at $0.8 \mathrm{eV})$; (2) a more compact feature marked with B in Fig. 9(b) (LUMO at $0.95 \mathrm{eV}$ ); and (3) a featureless structure marked with B' (LUMO at $1.10 \mathrm{eV}$ ) that is located in the plane of the domain wall DW1. The center positions of the molecules in the two sublattices A and B of the interface layer are indicated in Figs. 9(b) and 9(c), respectively, by open circles. As in the case of the surface layer, solid lines between circles correspond to translations by the fundamental unit cell vector $(10.6 \AA$ for both $\mathrm{A}$ and $\mathrm{B}$ ). Before discussing the three molecular species in the interface layer individually, we turn to the symmetry properties of the interface layer.

In Figs. 9(b) and 9(c) one clearly observes a local twofold rotational symmetry $R_{2}$ around the centers of any of the $\mathrm{B}^{\prime}$ molecules in the domain wall DW1. This symmetry is present both in the center positions of the A and B molecules as well as in the feature shapes of the individual species. For example, the bend in molecule A that is apparent in Fig. 9(b) (and that breaks the twofold symmetry $R_{2}$ of the image feature of the A molecule itself) is clearly rotated by $180^{\circ}$ if molecules on either side of DW1 are considered. A similar but less apparent symmetry relation is valid for B molecules on either side of DW1. In contrast the image features of $\mathrm{B}^{\prime}$ molecules posses $R_{2}$ symmetry, as must be, because they are located on the symmetry axis of $R_{2}$. The lateral shifts between B molecules on either side of DW1 relative to the $\mathrm{B}^{\prime}$ molecules in DW1 are also consistent with $R_{2}$. Interestingly, however, the sublattice of A molecules extends undisturbed across the DW1 domain wall.

It can therefore be concluded that DW1 separates two domains of the $\beta$-phase that are related by a $180^{\circ}$ rotation around an axis perpendicular to the surface. We label these two domains as $\mathbf{a}$ and $\mathbf{b}$. It is clear that the presence of a periodic array of $\mathbf{a}$ and $\mathbf{b}$ domains requires the existence of a second set of domain boundaries beside DW1. Looking at the images in Fig. 9, it is clear that this set, which we denote DW2, must be located beneath the molecular chains in the third layer.
Although we cannot directly see this set of domain boundaries, we do observe an axial shift along the $\langle 01 \overline{1}\rangle$-direction of the $\mathrm{A}$ and $\mathrm{B}$ (by approximately $1.5 \AA$ ) sublattices (but not the $\mathrm{C}$ sublattice) when crossing DW2. In contrast at the DW1 domain boundaries the $\mathrm{B}$ (by $1.5 \AA$ ) and $\mathrm{C}$ (by $3 \AA$ ) sublattices, but not the A sublattice, exhibit an offset along the $\langle 01 \overline{1}\rangle$-direction. The different behavior of the three sublattices $\mathrm{A}, \mathrm{B}$, and $\mathrm{C}$ at DW1 and DW2 shows that the latter two must be structurally nonequivalent. The nature of this difference will be discussed in more detail, but it is interesting to note that DW1 and DW2 seem to originate in the $\mathrm{C}$ and A sublattices, respectively, while sublattice $\mathrm{B}$, in which both types of domains boundaries can be found, seems to stitch the $\mathrm{A}$ and $\mathrm{C}$ sublattices together. This conjecture is consistent with the orientation of molecules in the B sublattice that will be subsequently discussed.

The alternating sequence of DW1 and DW2 defines the superstructure cell of the $\beta$-phase. The superstructure unit cell is oblique with translation vectors $\left|\mathbf{b}_{1}\right|=14 \AA$ along $\langle 01 \overline{1}\rangle$ and $\left|\mathbf{b}_{2}\right|=74 \AA$ at an angle of $72^{\circ}$ with respect to $\langle 01 \overline{1}\rangle$. This is close to what was predicted from the diffraction experiments 1 where seven fundamental unit cells make up the superstructure unit cell $(10.6 \AA \times 7 \approx 74 \AA)$. The overall periodicity in the $\langle 2 \overline{1} \overline{1}\rangle$-direction (i.e., perpendicular to the long axes of the molecules, not the molecular rows) is approximately $70.4 \AA$. On the basis of Fig. 9 we can count Tc molecules in the superstructure cell. It contains a total of at least 21 molecules, namely 6 molecules of type A, 6 of type B, 1 of type $\mathrm{B}^{\prime}$, 6 of type $C$, and 2 molecules in the chains (see further discussion). Moreover, there may be an additional molecule present beneath the chain in the DW2 domain boundary (see further discussion); this molecule, however, cannot be imaged in STM under any tunneling conditions.

\section{Structure model for the $\boldsymbol{\beta}$-phase}

We now discuss the position of individual molecules in the superstructure unit cell and derive a structure model for the $\beta$-phase. While the general structure and symmetry of the $\beta$-phase as discussed up to this point follows unambiguously from our STM data, the exact position and orientation of the various molecules in the superstructure cell cannot be pinned down by STM alone. Nevertheless, we can still obtain valuable hints toward a structure model from the STM images in Fig. 9.

The superstructure unit cell (containing 21 or even 22 Tc molecules) described previously requires a rather dense packing, namely 22 molecules in a total area of $985 \AA^{2}$, which corresponds to $44.8 \AA^{2}$ per molecule. This packing density cannot be achieved in a simple double-layer structure, since the most closely packed planar $\alpha$-phase has $100 \AA^{2}$ per molecule. The most compact double layer of flat-lying molecules would therefore require at least $50 \AA^{2}$ per molecule. On the other hand the $\beta$-phase appears in STM scans only $2.7 \AA$ thicker than the $\alpha$-phase. Therefore we conclude that at least some molecules in the $\beta$-phase are not parallel to the surface but tilted out of the surface plane. Analyzing the packing density layer by layer, we find that the basic structural unit of the interface layer, consisting of one A and one B molecule (as argued previously, the assignment of both $\mathrm{A}$ and $\mathrm{B}$ to the interface layer is based on their respective spectra), occupies an area of $141 \AA^{2}\left(\left|\mathbf{b}_{1}\right|=10.6 \AA,\left|\mathbf{b}_{2}\right|=14 \AA, \beta=72^{\circ}\right)$, 
i.e., $70.5 \AA^{2}$ per molecule. Since this is again considerably less than in the $\alpha$-phase, at least one of these molecules (A or B) should be tilted out of the interface plane to accommodate both of them (at least partially) in the interface layer. Such a tilt has already been predicted by Langner et al., ${ }^{1}$ but we can exclude one of their proposed models, namely the symmetric tilt of both molecules, since we observe these molecules to be nonequivalent in STM. Based on our STS data, ${ }^{35}$ we can conclude that the tilt of B molecules is larger than that of the A molecules, because the LUMO of A molecules is found closer to the Fermi level and is broadened more strongly; this reveals a stronger electronic coupling to the metal and hence a smaller tilt angle, if any.

It was already mentioned that Fig. 9(b) reveals differences between the image features corresponding to $\mathrm{B}$ and $\mathrm{B}^{\prime}$ molecules. This difference can be explained as a necessary consequence of, first, the tilt of B molecules and, second, the symmetry relation between $\mathbf{a}$ and $\mathbf{b}$ domains. Within the $\mathbf{a}$ and $\mathbf{b}$ domains, $\mathrm{B}$ molecules tilt in opposite directions (which causes the aforementioned contrast rotation of corresponding image features). $\mathrm{B}^{\prime}$ molecules, on the other hand, because they are located in the domain boundary DW1, by symmetry must be oriented with their short axis perpendicular to the surface. This is consistent with the electronic spectra, which indicate less screening by the metal for $\mathrm{B}^{\prime}$ molecules. Given the similar appearance of B and $\mathrm{B}^{\prime}$ molecules in Figs. 9(b) and 9(c) (except for the symmetry breaking of B molecules that was mentioned previously), we can further conclude that the out-of-plane tilt of $\mathrm{B}$ molecules is rather large but less than $90^{\circ}$.

The arrangement of $\mathrm{A}, \mathrm{B}$, and $\mathrm{B}^{\prime}$ molecules can be seen in the structure model of the $\beta$-phase displayed in Fig. $9(\mathrm{~d})$. While the positions of the $\mathrm{C}$ molecules relative to $\mathrm{A}$ and $\mathrm{B}$ (e.g., the slight displacement of $\mathrm{C}$ with respect to $\mathrm{A}$ in the direction $72^{\circ}$ off the $\langle 01 \overline{1}\rangle$ ) follow from the comparison of Fig. 9(a) with Figs. 9(b) and 9(c), the small tilt of the C molecules in the model of Fig. 9(d) is motivated solely by the herringbone arrangement in bulk tetracene; ${ }^{67}$ in the STM image of the surface layer in Fig. 9(a) a tilting asymmetry is not discernible.

The structure model shows the difference between domain walls DW1 and DW2: Across DW1 B molecules tilt away from each other, while they tilt toward each other across DW2. The structure model also shows a void in the interface layer at DW2, which we have filled with an additional molecule (shown in grey) that is not observable in STM. For symmetry reasons this molecule must be either perpendicular or parallel to the interface. Finally, the structure model reveals that by their almost upright orientation $\mathrm{B}$ and $\mathrm{B}^{\prime}$ molecules indeed transmit the registry between the $A$ and $C$ sublattices in the interface and surface layers, respectively. Therefore it is natural that sublattice B is disrupted by both types of domain walls.

It is apparent in Fig. 9 that the domain walls DW2 are decorated by molecular chains in the third layer. The chains are oriented along the $\langle 01 \overline{1}\rangle$-direction. It has been shown in Ref. 35 that chains may consist of monomers ( $\mathrm{D}^{\prime}$ molecule) or dimers ( $\mathrm{E}$ and $\mathrm{D}$ molecules), dimers being the more frequent case [cf. Fig. 9(d)]. The fact that chains appear only on one kind of domain boundary, namely DW2, once more reveals the structural difference between DW1 and DW2, in agreement with our structure model in Fig. 9(d). The structure model also reveals the reason for the formation of chains. Because
$\mathrm{B}$ molecules are tilted toward each other across DW2, there is no room for a $\mathrm{C}$ molecule within DW2. Hence, in DW2 the surface layer of the $\beta$-phase exhibits vacancies. The molecular chains apparently passivate these vacancies. In agreement with this argument, we have never observed patches of the $\beta$-phase devoid of chains; they are an integral part of the $\beta$-phase. In a sense the $\mathrm{D}^{\prime}$ molecule can be understood as a $\mathrm{C}$ molecule which is squeezed out of the surface layer by the inclination of B molecules in DW2. It is clear that there are two equivalent positions for the $\mathrm{D}^{\prime}$ monomer: one to the left and one to the right of the domain-wall axis. This rationalizes why the monomer molecule $\mathrm{D}^{\prime}$ is bistable. ${ }^{35}$ This instability is fixed if a second molecule, namely the E molecule, binds to the domain wall, thus forming a D-E dimer. While individual chain dimers do not switch any more, chain defects at which a short sequence of D-E dimers switches to E-D occur frequently. This is a consequence of the equivalence of the two configurations of the $\mathrm{D}^{\prime}$ molecule in DW2, which may both serve as nuclei for the formation of a dimer.

\section{Metastability of the $\beta$-phase}

Upon annealing at temperatures above $230 \mathrm{~K}$ and subsequent cooling below $230 \mathrm{~K}$, the $\beta$-phase transforms into the $\alpha$-phase. ${ }^{1}$ Thus, the $\beta$-phase is metastable. To study this transition by means of STM, we annealed the $\beta$-phase for 20 hours at room temperature. After subsequent cooling to $100 \mathrm{~K}$, a diffuse diffraction pattern of the $\alpha$-phase was observed [Figs. 10(a) and 10(b)], in agreement with Ref. 1.

The STM image displayed in Fig. 10(c) shows that apart from the dominant $\alpha$-phase that is responsible for the diffraction pattern, the local structure of the layer is quite rich. Extended regions with different molecular ordering are found. The most prominent minority phase is the $\gamma$-phase with its brick-wall structure that has been reported previously as a transition phase between the disordered low temperature $\delta$ phase and the $\alpha$-phase. Additionally, disordered regions as well as herringbone-like and rectangular molecular arrangements are observed, albeit less abundantly than the dominant $\alpha$ and $\gamma$-phases. Interestingly, at the beginning of the scanning process, we often observe separate Tc molecules on top of the converted layer that are left over from the surface layer of the $\beta$ phase, usually in more disordered regions of the ML. However, during scanning these molecules usually are swept away.

The local structure of the defect-rich Tc layer after annealing often changes during scanning at low temperature. An example is shown in Figs. 10(d) and 10(e). Comparing the two STM micrographs one recognizes a dislocation between two $\alpha$-phase domains (white-dashed arrows). Between these two scans the dislocation is displaced by one molecular row to the right. This is caused by a rigid shift to the left of one molecular row. Since the molecular pattern at the domain boundary is similar to the $\gamma$-phase (the corresponding unit cell and molecular centers are marked in blue), this shift can be interpreted as a $\gamma$ - to $\alpha$-phase transition at the border of the $\alpha$-phase domain on the right. This reorganization resembles the restructuring shown schematically in Fig. 6(c), thus providing experimental support for the mechanism of the $\gamma$ - to $\alpha$-phase transition suggested in Sec. IIIE. 

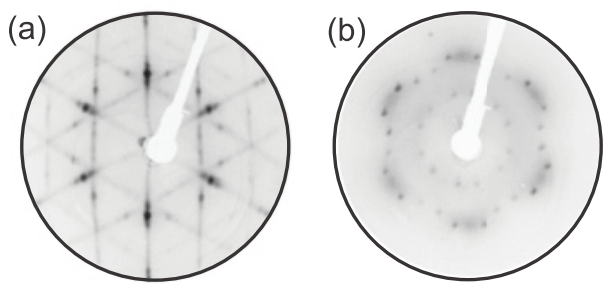

(c)

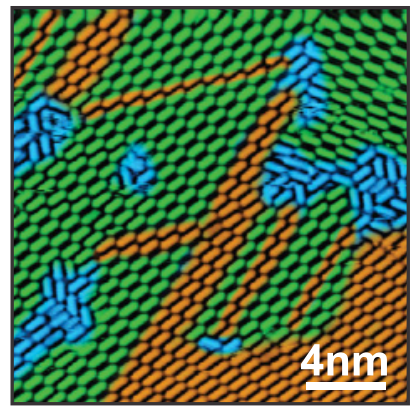

(d)

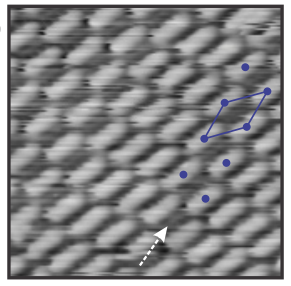

(e)

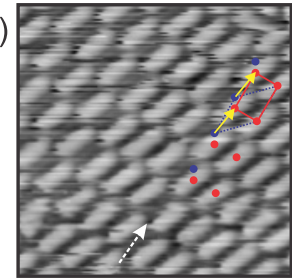

FIG. 10. (Color) (a)-(b) LEED patterns (beam energy $12 \mathrm{eV}$ ) of the $\mathrm{Tc} / \operatorname{Ag}(111)$ interface. Tc deposition at $\mathrm{T}=230 \mathrm{~K}$; Initial $\mathrm{Tc}$ coverage approximately 2.23 ML. (a) Before annealing. The pattern corresponds to the $\beta$-phase. (b) After annealing at RT and subsequent cooling to $100 \mathrm{~K}$. The pattern corresponds to the $\alpha$-phase. (c) STM image (bias voltage $0.4 \mathrm{~V}$, tunneling current $0.4 \mathrm{nA}$ ) of the molecular layer recorded after annealing, i.e. corresponding to the LEED pattern shown in (b). Areas with molecular arrangement corresponding to the $\alpha$-phase and $\gamma$-phase are shaded in orange and green, respectively. Disordered areas are marked in blue. (d)-(e) Two consecutive STM scans demonstrate a change of the local structure in the layer-the border between two neighboring translational domains of $\alpha$-phase (white arrows) moves to the left; this is effected by the rigid translation of a molecular row (as shown in Fig. 7(b)) to the right. Note that the molecular arrangement at the border agrees well with the structure of the $\gamma$-phase. Corresponding unit cells are marked in red and blue.

The transformation of the $\beta$-phase into the $\alpha$-phase happens because of the decomposition of the basic structural unit of the $\beta$-phase, i.e., the triplet of (partially tilted) $\mathrm{A} / \mathrm{B} / \mathrm{C}$ molecules, by annealing. Molecules that are left over after this restructuring (mostly $\mathrm{B}$ and $\mathrm{C}$ molecules) are mobile at room temperature and can either desorb or nucleate to form clusters on top of the $\alpha$-phase. The A molecules, which are released from their interaction with $\mathrm{B}$ and $\mathrm{C}$ molecules, may then establish a more direct bonding with the substrate.

\section{E. Driving force behind the formation of the $\beta$-phase}

We now turn to a discussion of the driving force behind the formation of the $\beta$-phase. To this end, we first compare the complex structural motif of the $\beta$-phase with the crystal structure of bulk Tc, which is shown in Fig. 9(d). The relative

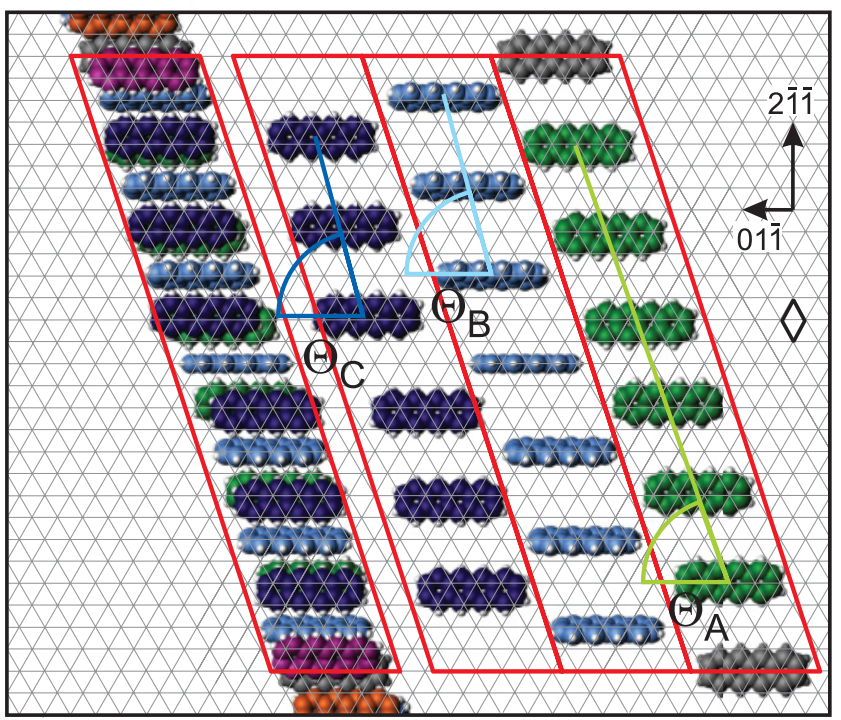

FIG. 11. (Color) Surface registry of the $\mathrm{Tc} / \operatorname{Ag}(111) \beta$-phase. Grey lines: lattice lines of $\operatorname{Ag}(111)$. Tc structure is shown as in Fig. 9(d). The angles $\Theta_{A}=71^{\circ}, \Theta_{B}=76^{\circ}$, and $\Theta_{C}=76^{\circ}$ are the orientations of molecular rows in the respective sublattices relative to the $\langle 01 \overline{1}\rangle$-direction of silver.

orientations of $\mathrm{B}$ and $\mathrm{C}$ molecules in the $\beta$-phase agree well with the angle between two nonequivalent molecules in bulk Tc. Moreover, the distance between two equivalent Tc molecules in the bulk crystal is $10.2 \AA$, which is close to the periodicity of $\mathrm{B}$ and $\mathrm{C}$ sublattices in the corresponding direction (10.6 and $10.9 \AA$ ). We can thus conclude that the arrangement of Tc molecules in the $\mathrm{B}$ and $\mathrm{C}$ sublattices of the $\beta$-phase resembles the structure of bulk Tc. At the same time, we have already seen that the A sublattice is to some extent influenced by the substrate and forced into a point-on-line coincidence with the substrate in which each $\mathrm{A}$ molecule is located on an atomic row of silver atoms (Fig. 11). It thus looks as if the $\beta$-phase of Tc is a pseudomorphic phase of $\mathrm{Tc}$ on $\operatorname{Ag}(111)$, in which the crystal structure of the molecular solid is modified at the interface in order to achieve a compromise between the molecular arrangement enforced by the substrate and the intrinsic bulk structure of the molecular solid.

In Fig. 11 we have plotted the registry of the sublattices $\mathrm{A}, \mathrm{B}$, and $\mathrm{C}$ with the $\operatorname{Ag}(111)$ surface separately. Note that the absolute positions of the molecules on the $\operatorname{Ag}(111)$ have been chosen arbitrarily, as these positions are not known experimentally. The relative positions, however, are those which have been deduced from the STM images in Fig. 9. The figure shows clearly that the tilt angles $\theta$ between the molecular rows and the $\langle 01 \overline{1}\rangle$-direction of the substrate (which coincides with the long molecular axes) is different for the $\mathrm{A}$ sublattice on the one hand $\left(71^{\circ}\right)$ and the $\mathrm{B}$ and $\mathrm{C}$ sublattices on the other $\left(76^{\circ}\right)$. The corresponding angle in bulk crystal structure of Tc is $78^{\circ}$. This shows two things. First, the different orientations of the three sublattices require the existence of domain boundaries along the $\langle 01 \overline{1}\rangle$-directions of the substrate, in which molecular rows are displaced along $\langle 01 \overline{1}\rangle$ in order to bring the sublattices back into registry. Second, we see that 
in going from sublattice A to sublattices $\mathrm{B}$ and $\mathrm{C}$, the angle $\theta$ becomes more bulk-like, supporting the idea that the $\beta$-phase is a pseudomorphic phase of bulk Tc.

It thus appears that the $\beta$-phase marks the beginning of heteroepitaxial growth of $\mathrm{Tc}$ in its native structure on $\mathrm{Ag}(111)$. This growth mode is remarkable because it implies that molecules in direct contact with the $\operatorname{Ag}(111)$ substrate are forced to rotate out of the plane of the surface. This is an unusual observation for the adsorption of large $\pi$-conjugated adsorbates on metal surfaces. Apparently in the competition between the molecule-molecule interaction, which according to our force-field calculations favors the edge-on-plane arrangement that is observed in the bulk over the mostly repulsive side-by-side arrangement of molecules in the surface plane (Fig. 7), and the molecule-substrate interaction, which favors planar arrangement of molecules in the surface plane, the intermolecular interaction prevails at least partially in that the most densely packed structure of flat-laying molecules ( $\alpha$-phase) is diluted, and tilted B molecules are interspersed into the first molecular layer at the substrate.

Unfortunately, the heteroepitaxy of $\mathrm{Tc}$ on $\operatorname{Ag}(111)$ cannot be extended beyond the 2.23 molecular layers of the $\beta$-phase. Apparently the strain in the $\beta$-phase is too large to allow a continued layer-by-layer growth. This is a common observation in heteroepitaxy: If the strain in the pseudomorphic layer is too large, pseudomorphic growth is discontinued, a dislocation plane occurs, and on top of this 3D islands grow in the relaxed bulk structure.

\section{CONCLUSION}

Using low temperature STM, we have studied the structure of the $\mathrm{Tc} / \mathrm{Ag}(111)$ interface in the coverage range 0 to $2.4 \mathrm{ML}$. We found single diffusing molecules, a disordered layer of mutually repelling molecules, a commensurately ordered lowdensity phase, a monolayer phase with point-on-line registry, and a point-on-line 2.23 ML phase, which appears to be a pseudomorphic version of the bulk structure of tetracene. The structural simplicity of the tetracene molecule and of the close-packed $\mathrm{Ag}(111)$ surface, contrasting with the rich phenomenology of the very different phases which we have observed, make $\mathrm{Tc} / \operatorname{Ag}(111)$ a suitable model system to study the complexities of organic semiconductor heteroepitaxy on metal surfaces.

\section{ACKNOWLEDGMENTS}

We thank M. Sokolowski (University of Bonn), B. Voigtländer (Forschungszentrum Jülich), G. Meyer (IBM Research, Zurich), and S. Fölsch (Paul-Drude-Institute, Berlin) for helpful discussion. This work was partially supported by the Deutsche Forschungsgemeinschaft (DFG) via priority program 1121 "Organic field effect transistors: structural and dynamic properties" and the project TA244. *s.subach@fz-juelich.de

${ }^{1}$ A. Langner, A. Hauschild, S. Fahrenhoz, and M. Sokolowski, Surf. Sci. 574, 153 (2005).

${ }^{2}$ C. Stadler, S. Hansen, I. Kröger, C. Kumpf, and E. Umbach, Nature Physics 5, 153 (2009).

${ }^{3}$ H. Marchetto, U. Groh, T. Schmidt, R. Fink, H. J. Freund, and E. Umbach, Chem. Phys. 325, 178 (2006).

${ }^{4}$ G. Hlawacek, P. Puschnig, P. Frank, A. Winkler, C. AmbroschDraxl, and C. Teichert, Science 321, 108 (2008).

${ }^{5}$ A. C. Dürr, F. Schreiber, K. A. Ritley, V. Kruppa, J. Krug, H. Dosch, and B. Struth, Phys. Rev. Lett. 90, 016104 (2003).

${ }^{6}$ F.-J. Meyer zu Heringdorf, M. C. Reuter, and R. M. Tromp, Nature 412, 517 (2001).

${ }^{7}$ S. C. B. Mannsfeld, K. Leo, and T. Fritz, Phys. Rev. Lett. 94, 056104 (2005)

${ }^{8}$ M. O. Blunt, J. C. Russell, M. d. C. Gimenez-Lopez, J. P. Garrahan, X. Lin, M. Schröder, N. R. Champness, and P. H. Beton, Science 322, 1077 (2008).

${ }^{9}$ C. Wagner, D. Kasemann, C. Golnik, R. Forker, M. Esslinger, K. Müllen, and T. Fritz, Phys. Rev. B 81, 035423 (2010).

${ }^{10}$ G. Gonella, H.-L. Dai, and T. J. Rockey, J. Phys. Chem. C 112, 4696 (2008).

${ }^{11}$ G. Mercurio, E. R. McNellis, I. Martin, S. Hagen, F. Leyssner, S. Soubatch, J. Meyer, M. Wolf, P. Tegeder, F. S. Tautz, and K. Reuter, Phys. Rev. Lett. 104, 036102 (2010).

${ }^{12} \mathrm{~S}$. Soubatch, R. Temirov, and F. S. Tautz, Phys. Status Solidi A 205, 511 (2008).
${ }^{13}$ I. Kröger, B. Stadtmüller, C. Stadler, J. Ziroff, M. Kochler, A. Stahl, F. Pollinger, T.-L. Lee, J. Zegenhagen, F. Reinert, and C. Kumpf, New J. Phys. 12, 083038 (2010).

${ }^{14}$ B. Stadtmüller, I. Kröger, F. Reinert, and C. Kumpf, Phys. Rev. B 83, 085416 (2011).

${ }^{15}$ S. C. B. Mannsfeld and T. Fritz, Phys. Rev. B 69, 075416 (2004).

${ }^{16}$ S. C. B. Mannsfeld and T. Fritz, Phys. Rev. B 71, 235405 (2005).

${ }^{17}$ D. E. Hooks, T. Fritz, and M. D. Ward, Adv. Mater. 13, 227 (2001).

${ }^{18}$ S. Mannsfeld and T. Fritz, Mod. Phys. Lett. B 20, 585 (2006).

${ }^{19}$ D. Kasemann, C. Wagner, R. Forker, T. Dienel, K. Müllen, and T. Fritz, Langmuir 25, 12569 (2009).

${ }^{20}$ L. Onsager, Ann. NY Acad. Sci. 51, 627 (1949).

${ }^{21}$ P. Jakob and D. Menzel, Surf. Sci. 220, 70 (1989).

${ }^{22}$ G. Koller, R. I. R. Blyth, S. A. Sardar, F. P. Netzer, and M. G. Ramsey, Surf. Sci. 536, 155 (2003).

${ }^{23}$ R. Ruiz, A. C. Mayer, G. G. Malliaras, B. Nickel, G. Scoles, A. Kazimirov, H. Kim, R. L. Headrick, and Z. Islam, Appl. Phys. Lett. 85, 4926 (2004).

${ }^{24}$ C. Ambrosch-Draxl, D. Nabok, P. Puschnig, and C. Meisenbichler, New J. Phys. 11, 125010 (2009).

${ }^{25}$ T. Djuric, T. Ules, H.-G. Flesch, H. Plank, Q. Shen, C. Teichert, R. Resel, and M. G. Ramsey, Cryst. Growth and Design 11, 1015 (2011).

${ }^{26}$ S. Schiefer, M. Huth, A. Dobrinevski, and B. Nickel, J. Am. Chem. Soc. 129, 10316 (2007). 
${ }^{27}$ R. B. Campbell, J. M. Robertson, and J. Trotter, Acta Cryst. 15, 289 (1962).

${ }^{28}$ T. Siegrist, C. Besnard, S. Haas, M. Schiltz, P. Pattison, D. Chernyshov, B. Batlogg, and C. Kloc, Adv. Mater. 19, 2079 (2007).

${ }^{29}$ S. R. Forrest, Chem. Rev. 97, 1793 (1997).

${ }^{30}$ M. Fendrich and J. Krug, Phys. Rev. B 76, 121302 (2007).

${ }^{31}$ D. Y. Zhong, M. Hirtz, W. C. Wang, R. F. Dou, L. F. Chi, and H. Fuchs, Phys. Rev. B 77, 113404 (2008).

${ }^{32}$ X. N. Zhang, E. Barrena, D. Goswami, D. G. de Oteyza, C. Weis, and H. Dosch, Phys. Rev. Lett. 103, 136101 (2009).

${ }^{33}$ P. Yannoulis, R. Dudde, K. H. Frank, and E. E. Koch, Surf. Sci. 189-190, 519 (1987).

${ }^{34}$ K. H. Frank, P. Yannoulis, R. Dudde, and E. E. Koch, J. Chem. Phys. 89, 7569 (1988).

${ }^{35}$ S. Soubatch, C. Weiss, R. Temirov, and F. S. Tautz, Phys. Rev. Lett. 102, 177405 (2009).

${ }^{36}$ M. Mohai, Surf. Interface Anal. 36, 828 (2004).

${ }^{37}$ O. Neucheva, R. Temirov, and F. S. Tautz (unpublished).

${ }^{38}$ J. Lagoute, K. Kanisawa, and S. Folsch, Phys. Rev. B 70, 245415 (2004).

${ }^{39}$ R. Duschek, F. Mittendorfer, R. I. R. Blyth, F. P. Netzer, J. Hafner, and M. G. Ramsey, Chem. Phys. Lett. 318, 43 (2000).

${ }^{40} \mathrm{H}$. Ishii and K. Seki, in Conjugated Polymer and Molecular Interfaces (Marcel Dekker, New York, 2002), p. 293.

${ }^{41}$ H. Yamane, D. Yoshimura, E. Kawabe, R. Sumii, K. Kanai, Y. Ouchi, N. Ueno, and K. Seki, Phys. Rev. B 76, 165436 (2007).

${ }^{42}$ S. Lukas, G. Witte, and C. Wöll, Phys. Rev. Lett. 88, 028301 (2001).

${ }^{43}$ J. Repp, F. Moresco, G. Meyer, K.-H. Rieder, P. Hyldgaard, and M. Persson, Phys. Rev. Lett. 85, 2981 (2000).

${ }^{44}$ P. Hyldgaard and M. Persson, J. Phys. Condens. Matter 12, L13 (2000).

${ }^{45}$ E. Bertel, Surf. Sci. 367, L61 (1996).

${ }^{46}$ P. Bartlett, R. H. Ottewill, and P. N. Pusey, Phys. Rev. Lett. 68, 3801 (1992).

${ }^{47}$ M. D. Eldridge, P. A. Madden, and D. Frenkel, Nature 365, 35 (1993).

${ }^{48}$ A. B. Schofield, Phys. Rev. E 64, 051403 (2001).

${ }^{49}$ D. Frenkel, J. Phys. Condens. Matter 6, A71 (1994).

${ }^{50}$ F. Saija, S. Prestipino, and P. V. Giaquinta, J. Chem. Phys. 113, 2806 (2000).

${ }^{51}$ D. Frenkel, J. Phys. Condens. Matter 6, A71 (1994).

${ }^{52}$ J. G. Berryman, Phys. Rev. A 27, 1053 (1983).
${ }^{53}$ G. D. Scott and D. M. Kilgour, J. Phys. D: Appl. Phys. 2, 863 (1969).

${ }^{54}$ C. Song, P. Wang, and H. A. Makse, Nature 453, 629 (2008).

${ }^{55} \mathrm{P}$. Schaaf, in Lecture Notes in Physics: 25 Years of Non-Equilibrium Statistical Mechanics, Vol. 445 (Springer, Berlin, 1995).

${ }^{56}$ J. Repp, G. Meyer, S. M. Stojkovic, A. Gourdon, and C. Joachim, Phys. Rev. Lett. 94, 026803 (2005).

${ }^{57}$ A. Kraft, R. Temirov, S. K. M. Henze, S. Soubatch, M. Rohlfing, and F. S. Tautz, Phys. Rev. B 74, 041402(R) (2006).

${ }^{58}$ Gaussian 03. Revision C.02, M. J. Frisch, G. W. Trucks, H. B. Schlegel, G. E. Scuseria, M. A. Robb, J. R. Cheeseman, J. Montgomery, J. A. T. Vreven, K. N. Kudin, J. C. Burant, J. M. Millam, S. S. Iyengar, J. Tomasi, V. Barone, B. Mennucci, M. Cossi, G. Scalmani, N. Rega, G. A. Petersson, H. Nakatsuji, M. Hada, M. Ehara, K. Toyota, R. Fukuda, J. Hasegawa, M. Ishida, T. Nakajima, Y. Honda, O. Kitao, H. Nakai, M. Klene, X. Li, J. E. Knox, H. P. Hratchian, J. B. Cross, V. Bakken, C. Adamo, J. Jaramillo, R. Gomperts, R. E. Stratmann, O. Yazyev, A. J. Austin, R. Cammi, C. Pomelli, J. W. Ochterski, P. Y. Ayala, K. Morokuma, G. A. Voth, P. Salvador, J. J. Dannenberg, V. G. Zakrzewski, S. Dapprich, A. D. Daniels, M. C. Strain, O. Farkas, D. K. Malick, A. D. Rabuck, K. Raghavachari, J. B. Foresman, J. V. Ortiz, Q. Cui, A. G. Baboul, S. Clifford, J. Cioslowski, B. B. Stefanov, G. Liu, A. Liashenko, P. Piskorz, I. Komaromi, R. L. Martin, D. J. Fox, T. Keith, M. A. Al-Laham, C. Y. Peng, A. Nanayakkara, M. Challacombe, P. M. W. Gill, B. Johnson, W. Chen, M. W. Wong, C. Gonzalez, and J. A. Pople, 2004.

${ }^{59}$ J. Li, W.-D. Schneider, R. Berndt, O. R. Bryant, and S. Crampin, Phys. Rev. Lett. 81, 4464 (1998).

${ }^{60}$ R. Paniago, R. Matzdorf, G. Meister, and A. Goldmann, Surf. Sci. 336, 113 (1995).

${ }^{61}$ J. Li, W.-D. Schneider, and R. Berndt, Phys. Rev. B 56, 7656 (1997).

${ }^{62}$ I. Kröger, B. Stadtmüller, C. Wagner, C. Weiss, R. Temirov, F. S. Tautz, and C. Kumpf, submitted (2011).

${ }^{63}$ R. A. Scott and H. A. Scheraga, J. Chem. Phys. 42, 2209 (1965).

${ }^{64}$ J. C. Slater and J. G. Kirkwood, Phys. Rev. 37, 682 (1931).

${ }^{65}$ S. R. Forrest and Y. Zhang, Phys. Rev. B 49, 11297 (1994).

${ }^{66}$ A. Bondi, J. Phys. Chem. 68, 441 (1964).

${ }^{67}$ J. Robertson, J. Trotter, and V. C. Sinclair, Acta Cryst. 14, 697 (1961).

${ }^{68}$ I. Horcas, R. Fernandez, J. M. Gomez-Rodriguez, J. Colchero, J. Gomez-Herrero, and A. M. Baro, Rev. of Sci. Instr. 78, 013705 (2007). 